\section{(3)}

RT NO. 3-GALCIT 101 ntract No. RU-293

April 1, 1959 - June 30,1959

\title{
FUNDAMENTAL STUDIES RELATING TO SYSTEMS ANALYSIS OF SOLID PROPELLANTS
}

\section{ENGINEERING LIBRARY}

\section{IECHMEN REPRI}

15 July 1959
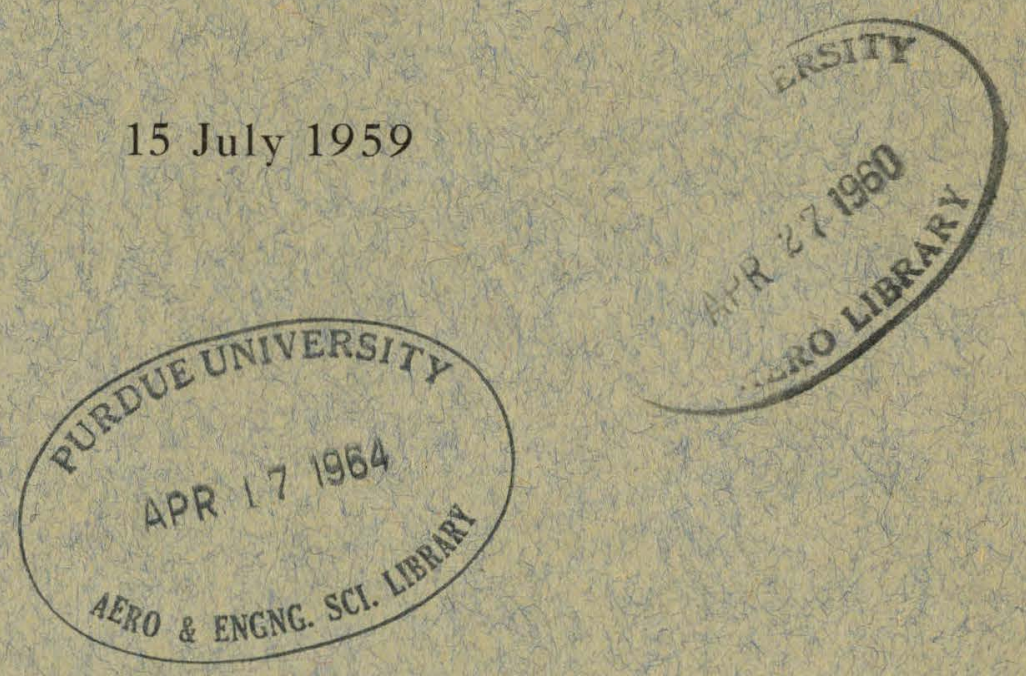

(2) Guggenheim Aeronatical Laboratory

(1) California Institute of Technology

Pasadena, California 
PROGRESS REPORT NO。3-GALCIT 101

Subcontract No. RU-293

April 1, 1959 - June 30, 1959

Fundamental Studies Relating to

Systems Analysis of Solid Propellants

15 July 1959

R.A. Schapery

L. D. Stimpson

M. L。Williams

This research is supported by The Thiokol Chemical Corp., Redstone Division

Guggenheim Aeronautical Laboratory

California Institute of Technology

Pasadena, California 
The earlier progress reports $(1,2)$ presented some essentials of model representation and a summary of some elastic solutions as preliminary material for viscoelastic analyses of solid propellants under various loading conditions. The present report is a continuation of the above with a brief section on Thermal Distributions, a section called Engineering Analysis, and one on Failure Criteria. The thermal distributions, obtained from heat transfer theory, are required for the thermoelastic formulations of section II ${ }^{(2)}$. The Engineering Analysis section includes several varied examples to assist in understanding the analysis techniques presented in the other sections. The final section. relates to mechanical failure of propellants and presents some preliminary thoughts as to how the study of this important problem area will be conducted.

Notation used throughout this report is defined within each section where used or consistent with that used in the previous reports and hence defined there. 


\section{TABLE OF CONTENTS}

I. Introduction to the Strain Analysis of Solid Propellant Grains $(1,2)^{\text {Page }}$

II. Elastic Solutions for Cylinders $(1,2)$

III. Temperature Distributions I

IV. Engineering Analysis 4

A. Example of Viscoelastic Pressure Stresses 5

B. Example of Viscoelastic Thermal Stresses 10

C. Example of Viscoelastic Behavior under Gravity 13

$\begin{array}{ll}\text { D. Stressed Grain Analysis } & 15\end{array}$

E. Composite Hollow Cylinder of $\mathrm{K}$ Layers under Internal Pressure

F. Displacements in a Rotating Hollow Cylinder under Internal Pressure and Axial Acceleration

V. Failure Criteria 


\section{TEMPERATURE DISTRIBUTIONS}

The thermal stress solutions given in the previous progress report ${ }^{(2)}$ require knowledge of the temperature distributions. Temperature changes in propellants are due almost exclusively to heat conduction and the (exothermic or endothermic) reactions during curing. Temperature change due only to straining is inconsequential, whereas radiation during long space flights may be more detrimental to the chemical composition of the propellant than in heating. At any rate, we shall first consider temperature distributions from the heat conduction equations reviewed in this section. Then the stresses and strains can be determined from equations presented in section II of the earlier report $(2)$ 。

While temperature distributions are generally transient in nature, the stresses and strains can usually be calculated, assuming the temperature distribution is pseudo-static, and creating instantaneous thermoelastic strains. Thus, as the temperature varies with time and space, we have thermal strains which also are time and space dependent. It was shown by Biot ${ }^{(3)}$ that the time effects in both the viscoelastic propellant and in the temperature can be included simultaneously in a precise way. This will be demonstrated in later articles through presentations of examples.

Particular mention is made of the Rohm and Has articles $(4)$ which include the thermoelastic strain formulations due both to heat transfer and curing reactions. In addition, incremental radial and axial casting are compared with the single casting procedure. Exotherms have been calculated by Nichols et al $(5,6)$, using a programed numerical integration of the heat conduction equations containing the chemical heat release term. 
HEAT TRANSFER EQUATION:

$$
\left.K\left(\frac{\partial^{2} T}{\partial r^{2}}+\frac{1}{r} \frac{\partial T}{\partial r}+\frac{1}{r^{2}} \frac{\partial^{2} T}{\partial \theta^{2}}+\frac{\partial^{2} T}{\partial z^{2}}\right)+Q_{1}=c \rho \frac{\partial T}{\partial t} \quad \text { (INFINITELY LONG }\right)
$$

RADIAL HEAT FLOW WITHOUT INTERNAL HEAT SOLRCES:

$$
k\left(\frac{\partial^{2} T}{\partial r^{2}}+\frac{1}{r} \frac{\partial T}{\partial r}\right)=\frac{\partial T}{\partial t}, \quad k \equiv \frac{K}{\rho C}
$$

TYPES OF BOUNDARY CONDITIONS:

1.) $T=T_{0}$,

2.) $\frac{\partial T}{\partial r}=0$,

3.) $\frac{\partial T}{\partial r}=$ CONST.,

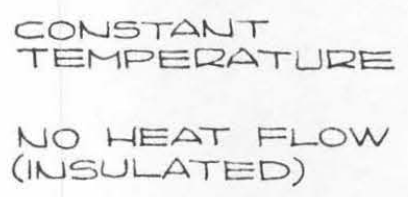

CONSTANT

(INSULATED)

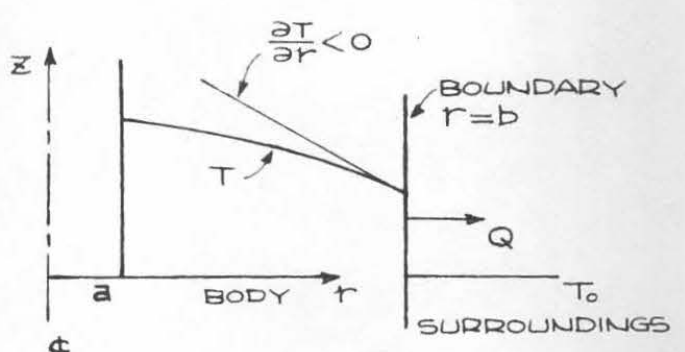

$\Phi$

PRESCRIBED FLOW RATE

4.) $K \frac{\partial T}{\partial r}+H\left(T-T_{0}\right)$ OR $\frac{\partial T}{\partial r}=h\left(T-T_{0}\right)$, RADIATION CONDITION,

$$
T_{0}=\text { TEMP. OF SURROUNDINGS, }
$$$$
Q=-2 \pi b K \frac{\partial T}{\partial r}=\text { HEAT FLUX/UNIT LENGTH }
$$

5.) $Q=2 \pi b \sigma E\left(T^{4}-T_{0}^{4}\right)$, BLACK BODY RADIATION, To ABSOLUTE

STEADY STATE RADIAL FLOW:

$$
\begin{aligned}
& T=T_{0}+\frac{T_{1}-T_{0}}{\log \left(\frac{b}{a}\right)} \log \left(\frac{b}{r}\right), \\
& T=T_{1}: \quad r=a \\
& T=T_{0}: \quad r=b \\
& Q=-2 \pi r k \frac{\partial T}{\partial r}=\frac{2 \pi k\left(T_{1}-T_{0}\right)}{\log \left(\frac{b}{a}\right)}
\end{aligned}
$$

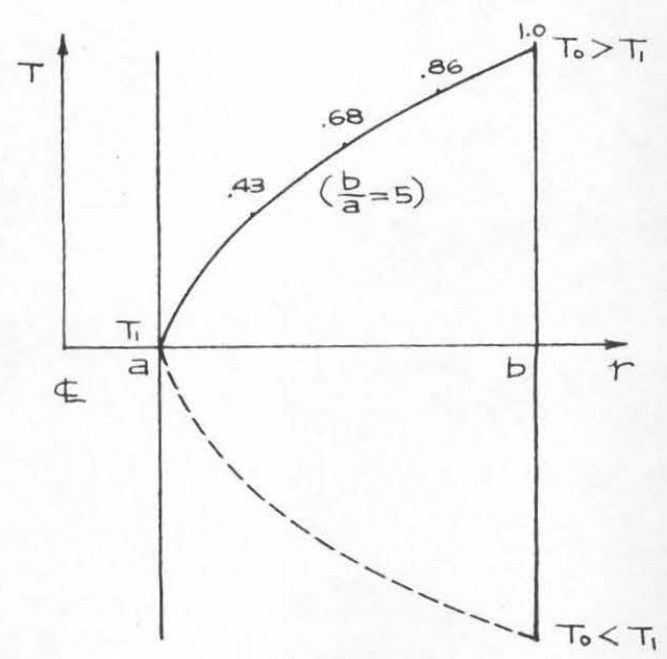


IRANSIENT RADIAL FLOW:

$$
k\left(\frac{\partial^{2} T}{\partial r^{2}}+\frac{1}{r} \frac{\partial T}{\partial r}\right)=\frac{\partial T}{\partial t}
$$

SEPARATING VARIABLES:

$$
\begin{aligned}
& T(r, t)=R(r) e^{-k \alpha^{2} t} \\
& \frac{d^{2} R}{d r^{2}}+\frac{1}{r} \frac{d R}{d r}+\alpha^{2} R=0, \text { BESSEL'S EQUATION }
\end{aligned}
$$

REFERENCE : 7 TEMDERATLRE INITIALLY ZERO, OUTSIDE TEMPERATLUDE SUDDENLY
RAISED TO TO:

$$
\begin{aligned}
T & =T_{0} \frac{\log \left(\frac{r}{a}\right)}{\log \left(\frac{b}{a}\right)} \\
& -\pi T_{0} \sum_{n=1}^{\infty} \frac{e^{-k \alpha_{n}^{2} t} J_{0}\left(a \alpha_{n}\right) J_{0}\left(b \alpha_{n}\right)}{J_{0}^{2}\left(a \alpha_{n}\right)-J_{0}^{2}\left(b \alpha_{n}\right)}\left[J_{0}\left(r \alpha_{n}\right) Y_{0}\left(a \alpha_{n}\right)-Y_{0}\left(r \alpha_{n}\right) J_{0}\left(a \alpha_{n}\right)\right]
\end{aligned}
$$

WHERE $\alpha_{n}$ ARE ROOTS OF :

$$
J_{0}(\dot{a} \alpha) Y_{0}(b \alpha)-Y_{0}(a \alpha) J_{0}(b \alpha)=0
$$

REFERENCE: 8

WHERE $N \cong Y, \quad R=\frac{b}{a}$ 


\section{ENGINEERING ANALYSIS}

This section includes several examples to indicate the procedures for solving various types of problems arising in the stress and strain analyses of solid propellant grains under various loading conditions. In order to demonstrate the analysis procedure, the examples chosen are purposely presented in simpler forms than that which might be required for realistic grain analyses. First, only the simplest of viscoelastic models are shown; second, the propellant is assumed incompressible, thus reducing the algebra considerably.

The first three examples demonstrate the use of the analogy between the viscoelastic and elastic states and deal with internal pressurization, thermal loading and gravity loading。

The second group of three examples in this section covers elastic analyses of grains bonded to cases. The first in this latter group covers the relative effects of case and propellant moduli upon each other and the possibilities of designing thinner cases. The second example covers multi-layered cylinders, e.g. grain, liner and case, under internal pressure and in particular is applied to the grain with thin liner and thin case.

The final example has been included to illustrate an approximate method of analysis currently under extensive study. This procedure is based upon a minimum energy prinçiple and the variational calculus. The problem treated is the axial elastic slump of a case bonded grain with free ends. 
A. Example of Viscoelastic Pressure Stresses

Consider an infinitely long cylindrical hollow grain enclosed by an elastic case. The elastic solution for an incompressible propellant $(\mathcal{\nu}=1 / 2)$ and a thin case under internal pressure in plane strain is $*$

$$
\begin{aligned}
& \sigma_{r}=-\frac{\left(\frac{b}{r}\right)^{2}-1+\frac{(3 / 2)}{1-\nu_{c}^{2}} \frac{h E_{c}}{b E}}{\left(\frac{b}{a}\right)^{2}-1+\frac{(3 / 2)}{1-\nu_{c}^{2}} \frac{h E_{c}}{b E}} p_{i} \\
& \sigma_{\theta}=\frac{\left(\frac{b}{r}\right)^{2}+1-\frac{(3 / 2)}{1-\nu_{c}^{2}} \frac{h E_{c}}{b E^{2}}}{\left(\frac{b}{a}\right)^{2}-1+\frac{(3 / 2)}{1-\nu_{c}^{2}} \frac{h E_{c}}{b E_{i}}} p_{i} \\
& \sigma_{z}=\frac{1-\frac{(3 / 2)}{1-\nu \nu_{c}^{2}} \frac{h E_{c}}{b}}{\left(\frac{b}{a}\right)^{2}-1+\frac{(3 / 2)}{1-\nu_{c}^{2}} \frac{h E_{c}}{b E}}
\end{aligned}
$$

Consider the Voigt model representation with isotropic material constants of a viscoelastic behavior which evinces a time lag in shear

$$
\tau_{i j}=\eta \frac{d \gamma_{i j}}{d t}+\mu \gamma_{i, j} \quad(i \neq j)
$$

In operational (Laplace) form with zero initial conditions,

$$
\bar{\tau}_{i j}=(\eta p+\mu) \bar{\gamma}_{i j}
$$

Note that for an infinitely rigid case $\left(\mathrm{hE}_{c} / \mathrm{bE}\right) \rightarrow \infty$ and $\sigma_{r}=\sigma_{\theta}=\sigma_{z}=p_{i}$,
or hydrostatic compression, consequently $\epsilon_{r}=\epsilon_{\theta}=\epsilon_{z}=0$ 
As from incompressible elasticity theory

$$
\tau_{i j}=\mu \gamma_{i j}=\frac{E}{3} \gamma_{i j}
$$

Comparing equations $(A-5)$ and $(A-6)$, we see the equivalent modulus becomes:

$$
E(P) \rightarrow 3(\eta P+\mu)
$$

This substitution for $\mathrm{E}$ is made into any of the initial equations to obtain the operational (viscoelastic) form for the respective stresses. Hence in $(A-1,2)$

$$
\bar{\delta}_{(r)}=-\frac{\left[ \pm\left(\frac{b}{r}\right)^{2}-1\right](\eta p+\mu)+\frac{h E_{c}}{2\left(1-\nu_{c}^{2}\right) b}}{\left[\left(\frac{b}{a}\right)^{2}-1\right](\eta p+\mu)+\frac{h E_{c}}{2\left(1-\nu_{c}^{2}\right) b}} \bar{p}_{i}(p)
$$

where upper sign applies for $\sigma_{r}$, and the lower for $\sigma_{\theta}$.

Approximating an igniter shock by assuming that the input pressure has an initially steep linear rise with time to ${ }^{{ }_{1}}$ followed by a constant pressure, one has

$$
\begin{array}{ll}
\bar{p}_{i}=\frac{k^{\prime}}{p^{2}} & \text { for } 0 \leqslant t<t_{1} \\
\bar{p}_{i}=\frac{p_{c}}{p} & \text { for } \quad t \geqslant t_{1}
\end{array}
$$

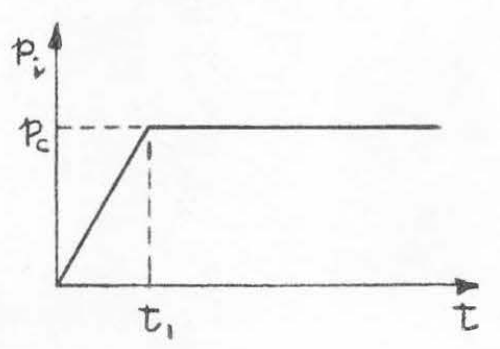

First, for $0 \leqslant \mathrm{t}<\mathrm{t}_{1}$,

$$
\bar{\sigma}_{(r)}=k \frac{p+a_{0}}{p^{2}(p+\alpha)}
$$

where

$$
k=k_{1} k^{\prime}=\left[-\frac{ \pm\left(\frac{b}{r}\right)^{2}-1}{\left(\frac{b}{a}\right)^{2}-1}\right] k^{\prime}, \quad k^{\prime}=\frac{p_{c}}{t_{1}}
$$




$$
\begin{aligned}
& a_{0}=\frac{\left[ \pm\left(\frac{b}{r}\right)^{2}-1\right] \mu+\frac{h E_{c}}{2\left(1-\nu_{c}^{2}\right) b}}{\left[ \pm\left(\frac{b}{r}\right)^{2}-1\right] \eta} \\
& \alpha=\frac{1}{\tau}=\frac{\left[\left(\frac{b}{a}\right)^{2}-1\right] \mu+\frac{h E_{c}}{2\left(1-\nu_{c}^{2}\right) b}}{\left[\left(\frac{b}{a}\right)^{2}-1\right] \eta}
\end{aligned}
$$

The Laplace inversion of $(\mathrm{A}-10)$ gives the time dependent physical stresses up to $t_{1}$ :

$$
\sigma_{\left(\begin{array}{l}
r \\
\theta
\end{array}\right)}=k \tau\left[a_{0} t+\left(1-a_{0} \tau\right)\left(1-e^{-\frac{t}{\tau}}\right)\right], \quad 0 \leqslant t<t_{1}
$$

and similarly for $t \geqslant t_{1}$ (including "initial" condition at $t_{1}$ ):

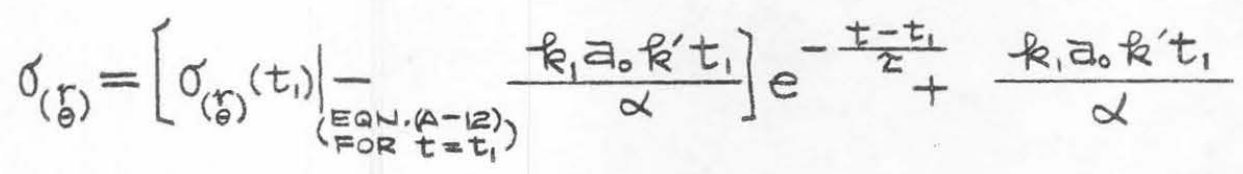

The strains and strain-rates are found from the stress-strain relationships:

$$
\begin{aligned}
& \epsilon_{\theta}=\frac{1}{E}\left[\sigma_{\theta}-\nu\left(\sigma_{r}+\sigma_{z}\right)\right] \\
& \epsilon_{z}=\frac{1}{E}\left[\sigma_{z}-\nu\left(\sigma_{r}+\sigma_{\theta}\right)\right]=0 \quad \text { (plain strain) }
\end{aligned}
$$

then

$$
\epsilon_{\theta}=\frac{(1+\nu)}{E}\left[(1-\nu) \sigma_{\theta}-\nu \sigma_{r}\right]
$$


using equations $(A-12)$ and $(A-13)$. The hoop strain on the inner surface for $0 \leqslant \mathrm{t}<\mathrm{t}_{1}$ becomes:

$\epsilon_{\theta_{r=a}}(t)=\frac{(1+\nu) k^{\prime}}{E}\left\{\frac{\left[\left(\frac{b}{a}\right)^{2}+1\right] \frac{\mu}{\eta}-\varepsilon_{c}^{\prime}+2 \nu\left(\varepsilon_{c}^{\prime}-\frac{\mu}{\eta}\right)}{\left[\left(\frac{b}{a}\right)^{2}-1\right] \frac{\mu}{\eta}+\varepsilon_{c}^{\prime}}\right\} t+\frac{2(1-\nu)^{2} k^{\prime}}{E} \frac{\varepsilon_{c}^{\prime}\left(\frac{b}{a}\right)^{2}}{\left\{\left[\left(\frac{b}{a}\right)^{2}-1\right] \frac{\mu}{\eta}+\varepsilon_{c}^{\prime}\right\}^{2}}\left(1-e^{\left.-\frac{t}{\tau}\right)}\right.$

and the strain-rate is simply the time derivative of $(A-16)$. For $t \geqslant t_{1}$ :

$$
\begin{aligned}
\epsilon_{\theta_{r=a}(t)=}= & \frac{1+\nu}{E}\left[( 1 - \nu ) \left(e ^ { - \alpha ( t - t _ { 1 } ) } \left\{\frac { k ^ { \prime } } { [ ( \frac { b } { a } ) ^ { 2 } - 1 ] ^ { \frac { \mu } { \eta } } + \varepsilon _ { c } ^ { \prime } } \left[\left(\left[\left(\frac{b}{a}\right)^{2}+1\right] \frac{\mu}{\eta}-\varepsilon_{c}^{\prime}\right) t_{1}\right.\right.\right.\right. \\
& \left.\left.\left.+\frac{2 \varepsilon_{c}^{\prime}\left(\frac{b}{a}\right)^{2}}{\left[\left(\frac{b}{a}\right)^{2}-1\right] \frac{\mu}{\eta}+\varepsilon_{c}^{\prime}}\left(1-e^{\left.-\frac{t_{1}}{\tau}\right)}\right]-k^{\prime} t_{1} \frac{\left[\left(\frac{b}{a}\right)^{2}+1\right] \frac{\mu}{\eta}-\varepsilon_{c}^{\prime}}{\left[\left(\frac{b}{a}\right)^{2}-1\right] \frac{\mu}{\eta}+\varepsilon_{c}^{\prime}}\right\}+k^{\prime} t_{1} \frac{\left[\left(\frac{b}{a}\right)^{2}+1\right]^{\frac{\mu}{\eta}-\varepsilon_{c}^{\prime}}}{\left[\left(\frac{b}{a}\right)^{2}-1\right] \frac{\mu}{\eta}+\varepsilon_{c}^{\prime}}\right]+\nu k^{\prime} t_{1}\right]
\end{aligned}
$$

and again the strain-rate is simply the time derivative of $(A-17)$ 。 Representative curves are shown in Fig. A-l for a rise time of onetenth of a second and an effective modulus of $10^{4}$ psi.

$$
\varepsilon_{c}^{\prime}=\frac{h E_{c}}{2\left(1-\nu_{c}^{2}\right) b \eta}
$$




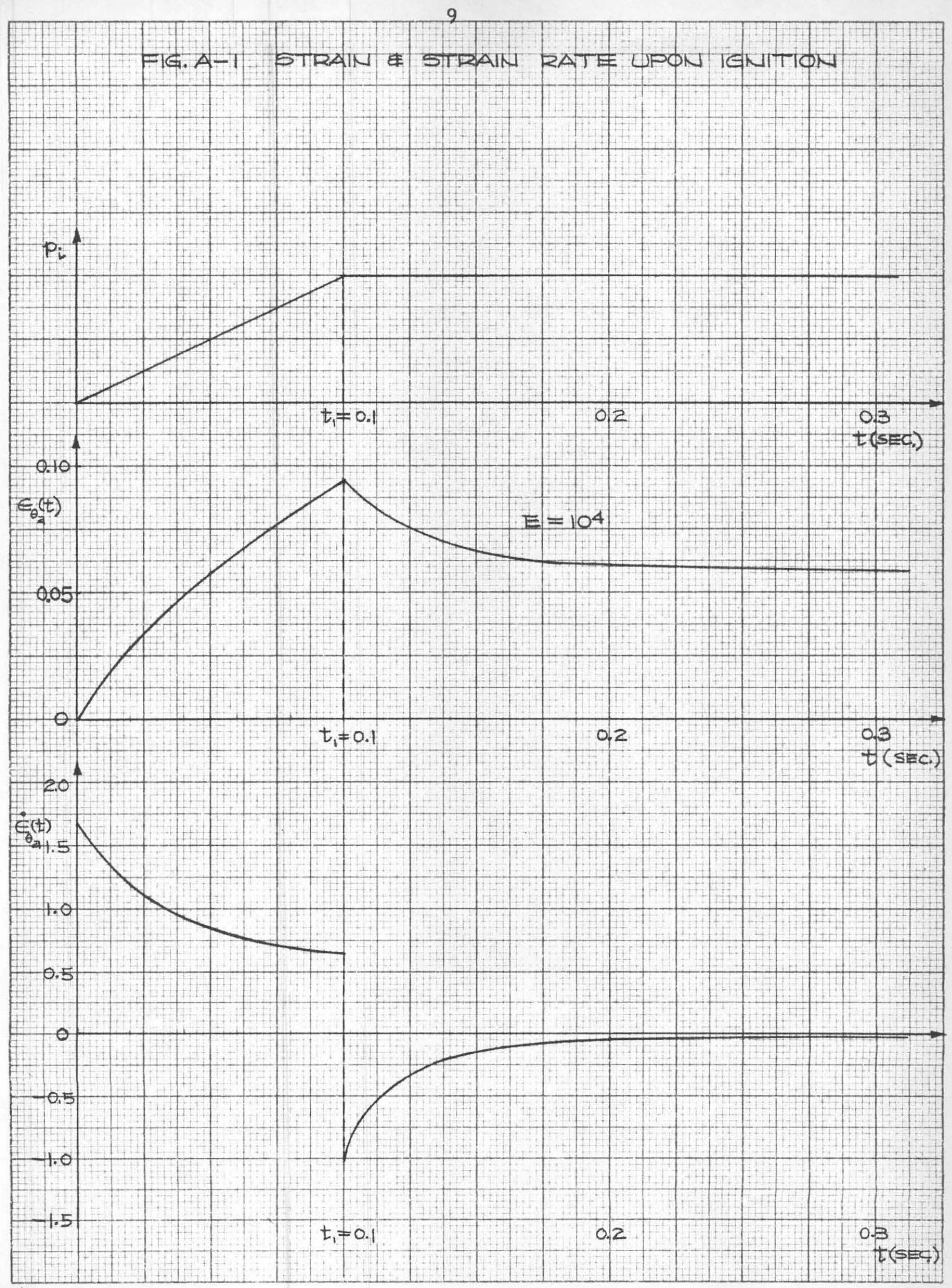


B. Example of Viscoelastic Thermal Stresses

Consider again the same cylindrical hollow grain with an elastic case. The elastic solution for the same uniform temperature rise of both the incompressible propellant and the thin case in plane strain is:

$$
\begin{aligned}
& \sigma_{r}=-p^{\prime} \frac{\left(1-\frac{a^{2}}{r^{2}}\right)}{\left(1-\frac{a^{2}}{b^{2}}\right)} \\
& \sigma_{\theta}=-p^{\prime} \frac{\left(1+\frac{a^{2}}{r^{2}}\right)}{\left(1-\frac{a^{2}}{b^{2}}\right)} \\
& \sigma_{z}=-p^{\prime} \frac{1}{\left(1-\frac{a^{2}}{b^{2}}\right)}
\end{aligned}
$$

where

$$
p^{\prime}=\frac{\left(\alpha-\alpha_{c}\right) E T}{\frac{3 a^{2}}{2\left(b^{2}-a^{2}\right)}+\left(1-\nu_{c}^{2}\right) \frac{b E}{h E_{c}}}
$$

Consider this time a Maxwell model representation of viscoelastic shear behavior:

$$
\frac{d \gamma_{i j}}{d t}=\frac{1}{\mu} \frac{d \tau_{i j}}{d t}+\frac{\tau_{i j}}{\eta} \quad(i \neq j)
$$

In operational (Laplace) form:

$$
p \bar{\gamma}_{i j}=\left(\frac{p}{M}+\frac{1}{\eta}\right) \bar{\tau}_{i j}
$$

From incompressible elasticity theory:

$$
\mu \gamma_{i j}=\frac{E}{3} \gamma_{i j}=\tau_{i j}
$$


By comparing equations $(B-6)$ and $(B-7)$, we see the equivalent modulus becomes in this case

$$
E(P) \rightarrow \frac{3 \eta \mu P}{\eta P+\mu}
$$

This substitution for $E(P)$ is made in equation $(B-4)$ to obtain the operational (viscoelastic) form for the bond pressure. (The coefficients of linear expansion are assumed independent of these.)

$$
-\bar{\sigma}_{r_{b}}(p)=\bar{p}^{\prime}(P)=\frac{\left(\alpha-\alpha_{c}\right) \eta \mu p=(P)}{\left[\frac{a^{2}}{2\left(b^{2}-a^{2}\right)}+\left(1-\nu_{c}^{2}\right) \frac{b \mu}{h E_{c}}\right] \eta p+\frac{a^{2} \mu}{2\left(b^{2}-a^{2}\right)}}
$$

Consider, as a simple temperature variation, a slow linear rise in temperature (somewhat compatible with the uniform temperature assumption):

$$
T=k^{\prime} t ; \quad \bar{T}(P)=\frac{k^{\prime}}{P^{2}}
$$

Substitution into $(B-9)$ gives

$$
\overline{P^{\prime}}(P)=\frac{k^{\prime}}{P\left(P+\frac{1}{\tau}\right)}
$$

where

$$
\begin{aligned}
& k=\frac{\left(\alpha-\alpha_{c}\right) \mu k^{\prime}}{\frac{a^{2}}{2\left(b^{2}-a^{2}\right)}+\left(1-\nu_{c}^{2}\right) \frac{b \mu}{h E_{c}}} \\
& \frac{1}{\tau}=\frac{\frac{a^{2} \mu}{2\left(b^{2}-a^{2}\right)}}{\left[\frac{a^{2}}{2\left(b^{2}-a^{2}\right)}+\left(1-\nu_{c}^{2}\right) \frac{b \mu}{h E_{c}}\right] \eta}
\end{aligned}
$$


The Laplace inversion of (B-11) gives again the simple lag function

$$
p^{\prime}(t)=\frac{k}{\alpha}\left(1-e^{-\frac{t}{\tau}}\right)
$$

From the initial equations:

$$
\begin{aligned}
& \sigma_{r}(t)=-\frac{k\left(1-\frac{a^{2}}{r^{2}}\right)}{\alpha\left(1-\frac{a^{2}}{b^{2}}\right)}\left(1-e^{-\frac{t}{\tau}}\right) \\
& \sigma_{\theta}(t)=-\frac{k\left(1+\frac{a^{2}}{r^{2}}\right)}{\alpha\left(1-\frac{a^{2}}{b^{2}}\right)}\left(1-e^{-\frac{t}{\tau}}\right) \\
& \sigma_{z}(t)=-\frac{k}{\alpha\left(1-\frac{a^{2}}{b^{2}}\right)}\left(1-e^{-\frac{t}{\tau}}\right)
\end{aligned}
$$


C. Example of Viscoelastic Behavior under Gravity

Consider a long hollow cylindrical grain supported by an elastically thick (rigid) case in an upright position. Neglecting end effects (or equivalently stated as an infinitely long cylinder) we have the grain subjected solely to one shear stress due to its own weight of:

$$
\tau_{r z}(r)=-\frac{\rho g}{2}\left(r-\frac{a^{2}}{r}\right)
$$

and a corresponding displacement (zero at case) of

$$
w(r)=-\frac{\rho \xi}{2 \mu}\left(a^{2}-\log \frac{b}{r}-\frac{b^{2}-r^{2}}{2}\right)
$$

Introducing now a more sophisticated three-element viscoelastic model such that

$$
\tau_{r} \frac{d \tau_{r z}}{d t}+\tau_{r z}=\mu_{1}\left[\tau_{\tau} \frac{d \gamma_{r z}}{d t}+\gamma_{r z}\right]
$$

where

$$
\begin{array}{r}
\tau_{\gamma}=\frac{\eta_{2}}{\mu_{z}}=\text { time constant for constant strain } \\
\tau_{\tau}=\eta_{2}\left(\frac{1}{\mu_{z}}+\frac{1}{\mu_{1}}\right)=\text { time constant for constant stress }
\end{array}
$$

Assuming an incompressible propellant $(\nu=1 / 2)$, the equivalent viscoelastic shear modulus becomes:

$$
\mu \rightarrow \frac{\mu_{1}\left(\tau_{\tau} P+1\right)}{\tau_{r} P+1}=\frac{\eta_{2}\left(\mu_{1}+\mu_{2}\right) P+\mu_{1} \mu_{2}}{\eta_{2} P+\mu_{2}}
$$

Substitution of equation $(C-4)$ into equation $(C-2)$, and considering a step input in gravity load, such as perhaps being suddenly uprighted, 


$$
\bar{w}(P)=-\frac{\rho}{2}\left(a^{2} \log \frac{b}{r}-\frac{b^{2}-r^{2}}{2}\right) \frac{g}{p} \frac{\eta_{2} p+\mu_{2}}{\eta_{2}\left(\mu_{1}+\mu_{2}\right) P+\mu_{1} \mu_{2}}
$$

or

$$
\bar{w}(P)=k \frac{P+a_{0}}{P\left(P+\frac{1}{\tau}\right)}
$$

where

$$
\begin{aligned}
& k=-\frac{\rho g}{2\left(\mu_{1}+\mu_{2}\right)}\left(a^{2} \log \frac{p}{r}-\frac{b^{2}-r^{2}}{2}\right) \\
& a_{0}=\frac{\mu_{0}}{\eta_{2}}=\frac{1}{\tau_{r}} \\
& \frac{1}{\tau_{\tau}}=\frac{\mu_{1} \mu_{2}}{\eta_{2}\left(\mu_{1}+\mu_{2}\right)}
\end{aligned}
$$

The Laplace inversion gives for the axial slump,

$w(t)=-\frac{\rho g a^{2}}{4 \mu_{1}}\left\{2 \log \frac{b}{r}-\left(\frac{b}{a}\right)^{2}\left[1-\left(\frac{r}{b}\right)^{2}\right]\right\} \frac{1+\left(\frac{\mu_{2}}{\mu_{1}}\right)-\left(\frac{\mu_{2}}{\mu_{1}}\right) e^{-\frac{t}{\tau_{2}}}}{1+\left(\frac{\mu_{2}}{\mu_{1}}\right)}$

where it may be noted that for long time, the elastic situation is approached, and depends only upon the static material modulus $\mu$ 


\section{Stressed Grain Analysis *}

The end-bonded (axially-stressed) elastic solutions presented in section II ${ }^{(2)}$ for pressure and temperature loading have some immediate applications aside from viscoelastic analyses. One is the estimation of bond stresses both along the cylindrical case and at the ends due to thermal shrinkage in cooling. Another interesting possibility is in the application of higher modulus propellants which would share the loads with the case. This requires a regressive pressuretime program for minimum case weight, allowing the case and propellant to share load initially and with the case alone at the end of burning. An increase in initial-to-final pressure ratio is possible through combining changes in grain geometry, various burning rates, higher pressure-area exponent $n$, erosive burning of the grain, and possibly nozzle throat erosion too. This means the case thickness can be reduced accordingly.

Figures D-1, 2, and 3 present the inner surface stresses and strains due to internal pressure for various web thicknesses (cylindrical grains) and case-to-propellant modulus ratios. The parameter $\varepsilon_{C}$ is a natural quantity arising from the analysis which relates the case modulus to propellant modulus weighted by their cross-sectional areas:

$$
\varepsilon_{c}=\frac{2 \pi b h E_{c}}{\pi\left(b^{2}-a^{2}\right) E}
$$

Present day motor designs result in values for $\varepsilon_{c}$ of the order of a thousand or greater. Smaller values of $\varepsilon_{c}$ are equivalent to thinner cases, lower modulus cases, or higher modulus propellants.

The author is indebted to J. I. Shafer for suggesting this investigation. 


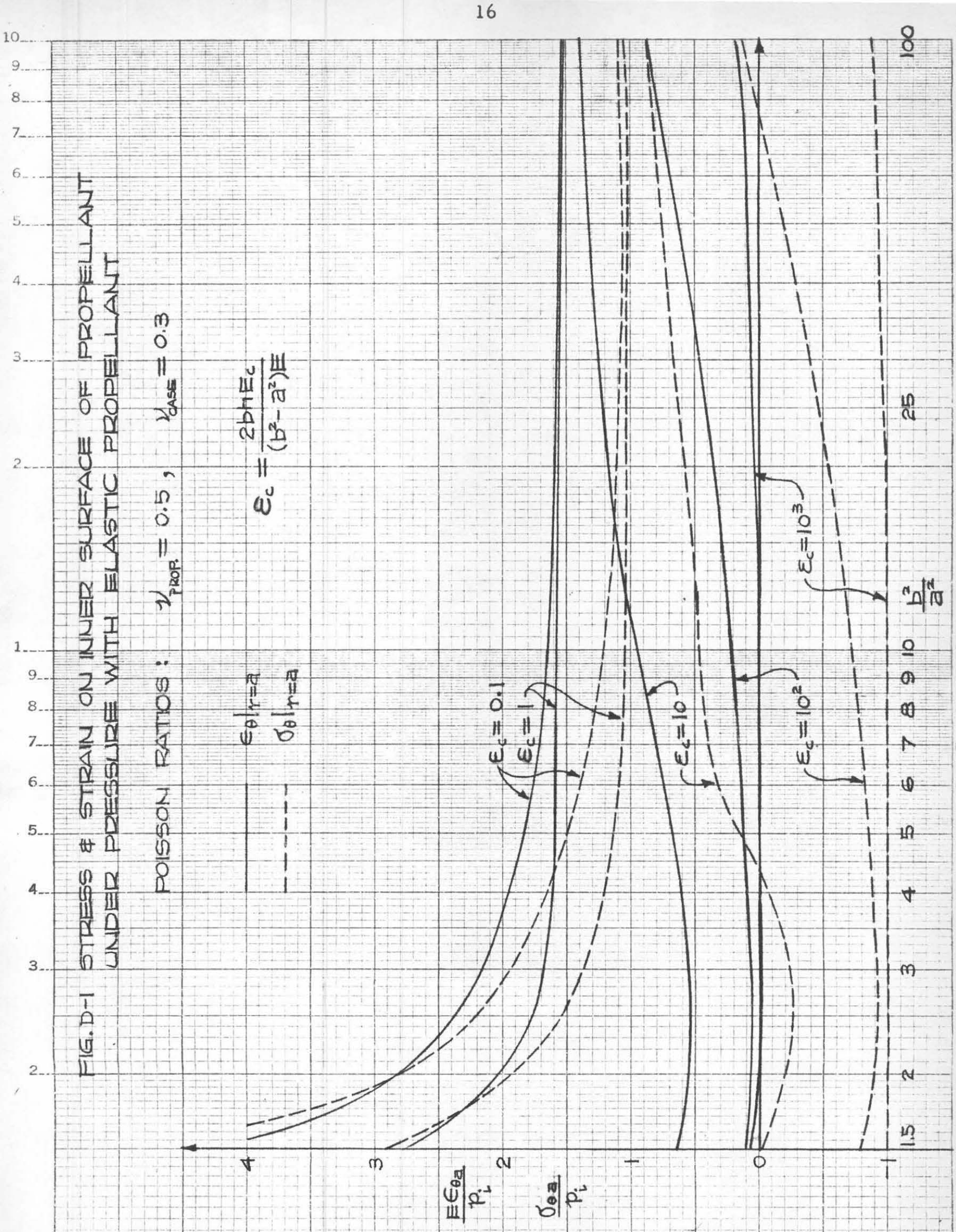




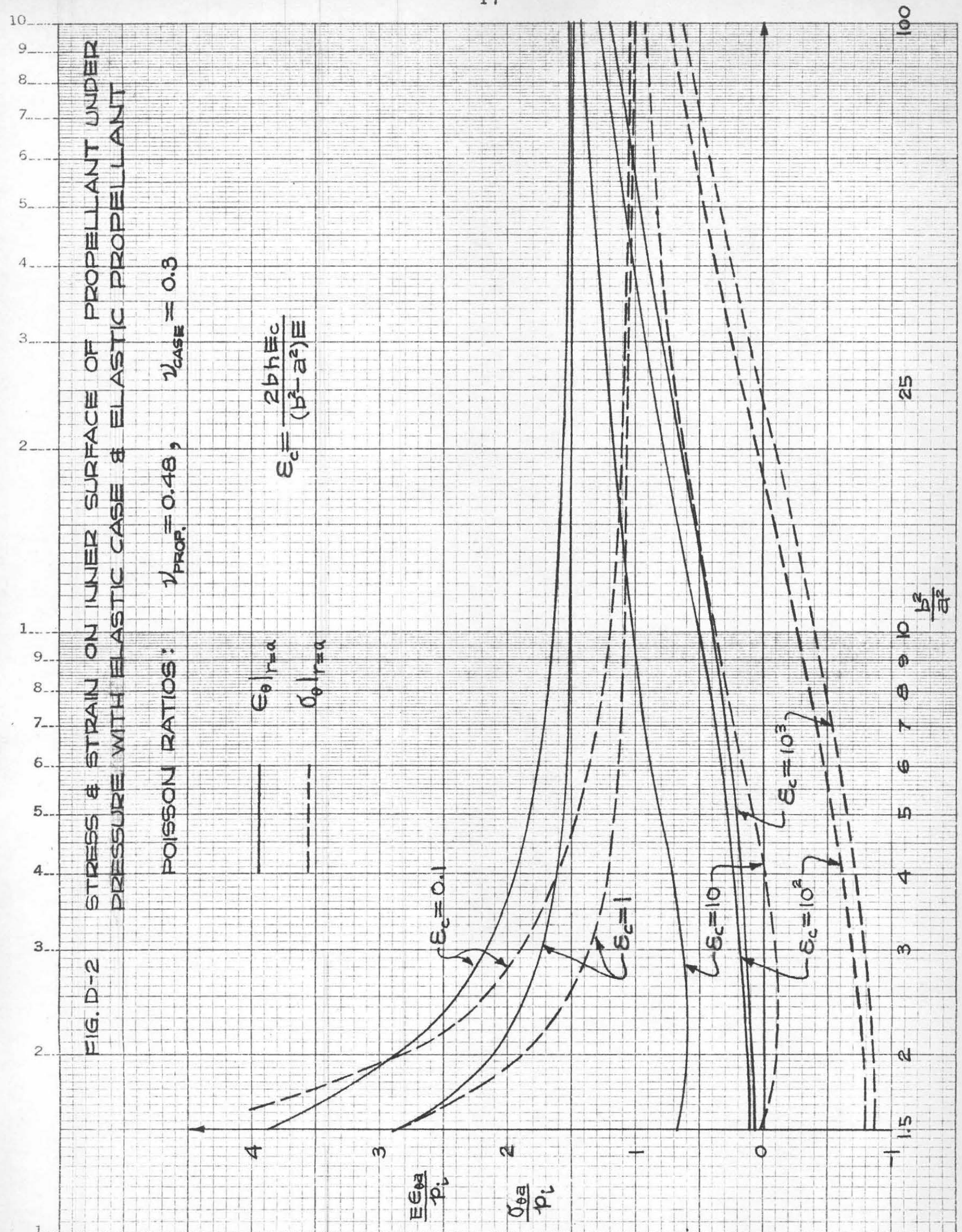




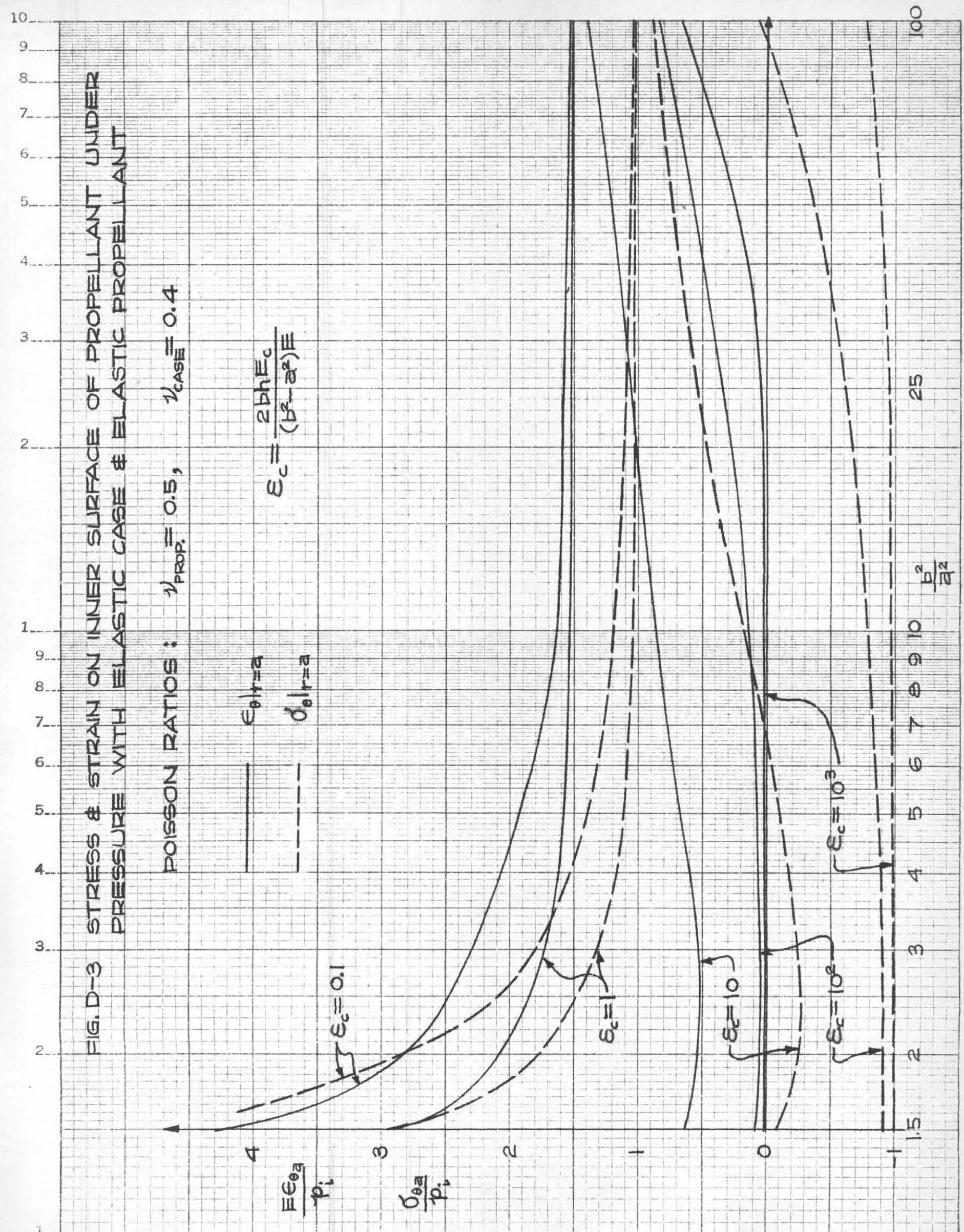


E. Composite Hollow Cylinder of $\mathrm{K}$ Layers under Internal Pressure

Inasmuch as it is often of interest to analyze a multi-layered concentric geometric, and hence be able to study the inclusion of a liner between the grain and case, or a radial incrementally cast grain, some elastic analyses pertaining to this case are summarized".

Consider the $n$th layer of a long right circular cylinder where we let $r_{n}$ denote inner radius, $p_{n}$ pressure at $r_{n}, r_{n+1}$ denote outer radius, $p_{n+1}$ pressure at $r_{n}+1^{\circ}$

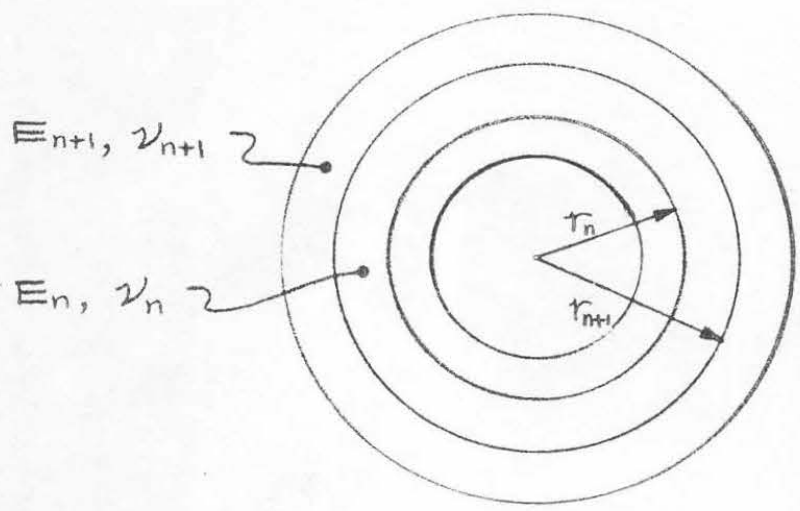

For plane stress the general solution of the displacement equilibrium equation is

$$
u_{n}(r)=A_{n} r+\frac{B_{n}}{r}
$$

where

$$
\begin{aligned}
& A_{n}=\frac{1-\nu_{n}}{E_{n}} \frac{P_{n} r_{n}^{2}-P_{n+1} r_{n+1}^{2}}{r_{n+1}^{2}-r_{n}^{2}} \\
& B_{n}=\frac{1+\nu_{n}}{E_{n}} \frac{r_{n}^{2} r_{n+1}^{2}\left(P_{n}-P_{n+1}\right)}{r_{n+1}^{2}-r_{n}^{2}}
\end{aligned}
$$

Contributed by Prof. K. S. Pister (University of California) from a more comprehensive investigation currently under study. 
The $\mathrm{k}-1$ continuity conditions at the internal boundaries together with the stress boundary conditions at the external boundaries are sufficient to determine the constants $A_{n}, B_{n}$ for each cylinder in a given case for alternatively, the internal boundary pressures). The continuity conditions are of the type

$$
u_{n}=u_{n+1} \quad \text { at } \quad r=r_{n+1}, \quad n=1,2, \cdots, k-1
$$

Combining equations $(E-1),(E-2)$ and substituting in $(E-3)$ leads to the result:

$$
L_{n} P_{n}+M_{n} P_{n+1}+N_{n} P_{n+2}=0, n=1,2, \cdots, k-1
$$

where

$$
\begin{aligned}
L_{n} & =\frac{2 r_{n}^{2}}{E_{n}}\left(r_{n+2}^{2}-r_{n+1}^{2}\right) \\
-M_{n} & =\left[\left(\frac{1-\nu_{n}}{E_{n}} r_{n+1}^{2}+\frac{1+\nu_{n}}{E_{n}} r_{n}^{2}\right)\left(r_{n+2}^{2}-r_{n+1}^{2}\right)+\left(\frac{1-\nu_{n+1}}{E_{n+1}} r_{n+1}^{2}+\frac{1+\nu_{n+1}}{E_{n+1}} r_{n+2}^{2}\right)\left(r_{n+1}^{2}-r_{n}^{2}\right)\right] \\
N_{n} & =\frac{2 r_{n+2}^{2}}{E_{n+1}}\left(r_{n+1}^{2}-r_{n}^{2}\right)
\end{aligned}
$$

To determine stresses and/or displacements in the " $n$ " th layer, obtain $P_{n}, P_{n+1}$ from the solution of equation $(E-4)$ and use in conventional cylinder equations, setting $P_{i}=P_{n}$ and $P_{0}=P_{n}+1^{*}$ For plane strain replace $E_{n}$ by $E_{n} / 1-\nu{ }_{n}^{2}$ and $\nu_{n}$ by $\nu_{n} / 1-\nu_{n}$.

Example: Internal pressure in three-laye $r$ cylinder

$$
\left.\begin{array}{l}
P_{1}=P_{i} \\
P_{4}=0
\end{array}\right\} \quad \text { External body conditions }
$$

Setting $\mathrm{n}=1,2$ in equation $(E-4)$ gives (using above conditions)

$$
\begin{aligned}
L_{1} P_{i}+M_{1} P_{2}+N_{1} P_{3} & =0 \\
L_{2} P_{2}+M_{2} P_{3} & =0
\end{aligned}
$$


whose solution is

$$
P_{2}=\frac{-L_{1} M_{2} P_{i}}{M_{1} M_{2}-L_{2} N_{1}} \quad, \quad P_{3}=\frac{L_{1} L_{2} P_{i}}{M_{1} M_{2}-L_{2} N_{1}}
$$

$L, M, N$ can be evaluated from equation $(E-5)$.

Pressures $P_{i}, P_{2}, P_{3}$ can be used in equations given in progress report 1 for the determination of stresses and displacements in the layers of the cylinder. In view of the cumbersome algebra involved, results will be given explicitly only for the instance in which the outer two layers are thin.

\section{Thin Liner and Thin Case}

$$
\begin{aligned}
& \frac{h_{l}}{b} \ll 1, \quad \frac{h_{c}}{b} \ll 1, \quad \lambda=\frac{b}{a} \\
& P^{\prime}=\text { interface pressure at } \quad r=b
\end{aligned}
$$

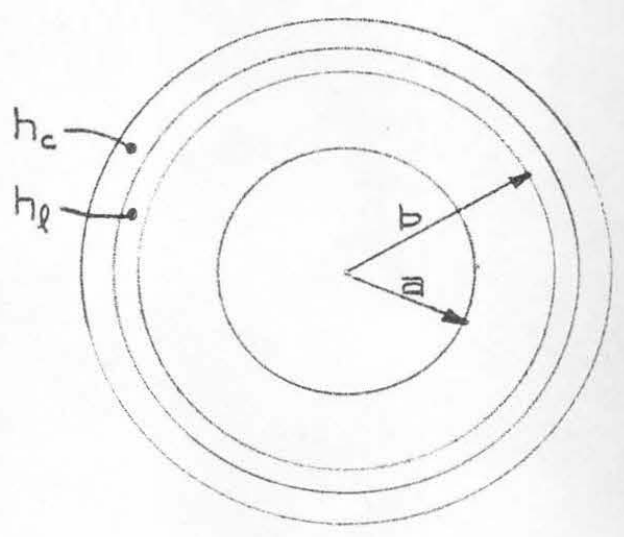

$\underline{\text { Plane Strain }}$

$$
P^{\prime}=\frac{2 P_{i}(1-\nu)}{\left[1+(1-2 \nu) \lambda^{2}+\frac{\left(\lambda^{2}-1\right)}{\frac{h_{c} E_{c}}{b E}\left(\frac{1+\nu}{1-\nu_{c}^{2}}\right)+\frac{h_{l} E_{l}}{b E}\left(\frac{1+\nu}{1-\nu_{c}^{2}}\right)}\right]}
$$

$\left\{\begin{array}{l}\text { Stresses } \\ \text { Strains }\end{array}\right\} \quad$ in cylinder, same as P-12, P-14 in Ref。1, using above value of $\mathrm{P}^{1}$.

Stress in case:

$$
\sigma_{\theta}=\frac{P^{\prime}}{\frac{h_{c}}{b}+\frac{h_{\ell}}{b} \frac{E_{\ell}}{E}}
$$

Stress in liner:

$$
\sigma_{\theta}=\frac{P^{\prime}}{\frac{h_{l}}{b}+\frac{h_{c}}{b} \frac{E_{c}}{E_{l}}}
$$


Discussion of Results: As can be seen from the expression for $P$ on the previous page, the presence of the liner does not affect the stress distribution in the grain, since the dominating term $h_{c} E_{c} / b E$ causes the fraction $\left(\lambda^{2}-1\right) /[<]$ to vanish for typical geometry and materials. Accordingly, the liner is of no significance pressurewise, as long as the ratio $h_{c} E_{c} / b E$ remains large, as it will for metal cases. In the event that non-metal cases with significantly lower moduli are used, or if grain stiffness were increased, the liner could become important in determining stress distribution due to pressure. 
$\underline{\text { F. Displacements in a Rotating Hollow Cylinder under Internal }}$ Pressure and Axial Acceleration

\section{Summary}

This paper presents an approximate solution for the displacements in the elastic range of a right circular cylinder, spinning about its axis of symmetry, subjected to internal pressure and axial acceleration. The solution is obtained by the method of restricted variation, making use of the potential energy theorem.

An attempt ${ }^{(9)}$ was made to use the Lagrange multiplier method to obtain zero integrated stresses on the interior cylindrical surface, without guarantee for the increase or decrease of the potential energy. For the case of axial acceleration loading, however, it is found that the use of the multiplier method leads to a poorer solution at the cost of satisfying one boundary condition (The multiplier method yields the solution of the infinitely long cylinder ${ }^{(2)}$.).

Because of the complexity of the problem, only the axial acceleration load is considered in detail, and graphical information given for a radius ratio of $1 / 2$, a length to outer diameter ratio of 1.25 , and Poisson's ratio of $\quad \boldsymbol{\nu}=0$ 。 For very long cylinders it is shown that the effect of finite length is localized at the ends of the cylinder.

The elastic solution does not exhibit the undesirable constriction at the aft end of the port opening; it is found that this opening widens under acceleration. However, if the aft end is rigidly supported, preventing any displacement of the surface, it is expected that a constriction occurs near the aft end. Whereas the support at the aft end may cause - excessive port narrowing, removal of the support will allow the cylinder to tear, due to excessive stresses; therefore an elastic support seems to be a good compromise.

* This presentation is an abbreviated form of Reference 9. 


\section{Notation}

\section{4}

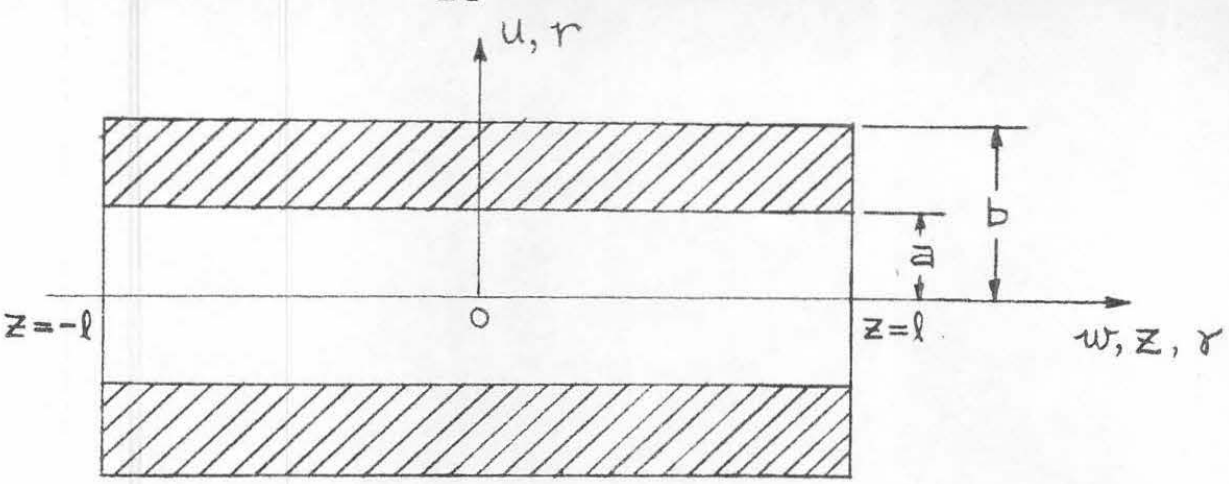

$\xi \quad=\frac{r}{b} \quad x=\frac{a}{b} ; t=\frac{z}{b} \quad \tau=\frac{l}{b}$

j $=$ axial body force

$\Omega=$ radial body force

$\omega=$ spin velocity

$\mathrm{p} \quad=$ pressure

$\mu=$ shear modulus

$\lambda=$ Lame constant

$\nu=$ Poisson's ratio $^{\prime}$

$v, v_{2}=$ first and second strain invariants

Solution of the Problem

The Potential Energy: In the absence of temperature effects, the potential energy is defined as

$$
U=W_{s}-W_{F}
$$




$$
\begin{aligned}
& W_{s}=\frac{1}{2} \int_{V O L}\left\{(\lambda+2 \mu) v^{2}-4 \mu v_{2}\right\} d(\text { VOL }) \\
& W_{F}=\int_{V O L}\{\gamma \omega+\Omega u+u(a) P(z)\} d(V O L)
\end{aligned}
$$

If product type functions are assumed for both $u$ and $w$, i. e.

$$
\begin{aligned}
& u=R_{1}(r) g(z) \\
& w=R_{2}(r) f(z)
\end{aligned}
$$

one finds for the potential energy

$\frac{u}{\pi}=\int_{-l}^{l}\left\{k g^{2}+L f^{2}+M f^{\prime \prime 2}+N g^{\prime 2}+P g^{\prime} f+Q g f^{\prime}+S f+\otimes g-J P g\right\} d z(F-6)$

The constants

$$
K L M \cdots-.-J
$$

are given in the appendix.

The Differential Equations: Performing the variation on $U$ and upon setting the variation equal to zero, one obtains the following relationships, if $M, N \neq 0$

$$
\begin{aligned}
& g^{\prime \prime}-\frac{K}{N} g=\frac{Q-P}{2 N} f^{\prime}+\frac{\Theta}{2 N} \\
& f^{\prime \prime}-\frac{L}{M} f=\frac{P-Q}{2 M} g^{\prime}+\frac{S}{2 M} \\
& 2 N g^{\prime}+P f=0 \quad \text { at } \quad z= \pm l
\end{aligned}
$$




$$
2 M f^{\prime}+Q g=0 \quad \text { at } \quad z= \pm l
$$

Upon defining the operator

$$
\mathcal{L}=\left\{\frac{d^{4}}{d z^{4}}-\left[\frac{K}{N}+\frac{L}{M}-\frac{(Q-P)^{2}}{4 M N}\right] \frac{d^{2}}{d z^{2}}+\frac{K L}{M N}\right\}
$$

it is found that

$$
\begin{aligned}
& \mathcal{L} g=-\frac{L \oplus}{2 M N} \\
& \mathcal{L} f=-\frac{K S}{2 M N}
\end{aligned}
$$

Solutions:

$$
\begin{aligned}
& g=a_{1} \sinh \lambda_{1} z+a_{2} \cosh \lambda_{1} z+a_{3} \sinh \lambda_{2} z+a_{4} \cosh \lambda_{2} z-\frac{\Theta}{2 k} \\
& f=\alpha_{1}\left[a_{1} \cosh \lambda_{1} z+a_{2} \sinh \lambda_{1} z\right]+\alpha_{2}\left[a_{3} \cosh \lambda_{2} z+a_{4} \sinh \lambda_{2} z\right]-\frac{s}{2 L}
\end{aligned}
$$

where

$$
\alpha_{1,2}=\frac{(P-Q) \lambda_{1,2}}{M \lambda_{1,2}^{2}-L}
$$

and $\lambda_{1,2}$ the solution of $\mathcal{L}(\lambda)=0$

The boundary conditions (9) and (10) yield the constants $a_{1}, a_{2}, a_{3}$, and $\dot{a}_{4}$ as defined in the appendix.

The Displacement Functions: $R_{1}$ is determined from the radial displacement in an infinitely long cylinder, spinning about its axis and $v$ interval pressure:

$$
R_{1}=\left[\frac{1}{\xi}-\xi\right](1-A)+B \xi\left[1-\xi^{2}\right]
$$




$$
A=\frac{\rho \omega^{2} b^{2}}{8 p^{*}}\left[(3+\nu) x^{2}-\frac{1}{1-\nu}\right] x^{2}
$$

$$
B=\frac{\rho \omega^{2} b^{2}}{8 P^{*}}\left[\frac{1}{1+\nu}-\frac{x^{2}}{1-\nu}\right]
$$

where

$$
\begin{array}{ll}
P^{*}=P & \text { if } \\
P^{*}=2 M(1+\nu) \text { if } & P \equiv 0
\end{array}
$$

arising from the normalization with respect to the pressure. The infinite cylinder under axial acceleration yields

$$
w_{w}=\frac{8 b^{2}}{4 \mu}\left\{1-\xi^{2}+2 x^{2} \ln \xi\right\}
$$

hence

$$
R_{2}=1-\xi^{2}+2 x^{2} \ln \xi
$$

The Special Case $p=w=0:$ Even though, in this case, the radial variation $R_{1}$ would be zero, it is felt that the radial displacement attenuates to zero in a fashion similar to the law

$$
R_{1}=\frac{1}{\xi}-\xi
$$

At least this function has a sounder physical basis than a simple parabolic law, hence

$$
\begin{aligned}
& u=\left(\frac{1}{\xi}-\xi\right) g(z) \\
& w=\left(1-\xi^{2}+2 x^{2} \ln \xi\right) f(z)
\end{aligned}
$$

Numerical Results: Numerical calculations for the configuration $x=1 / 2 \quad \nu=0 \quad \ell / b=1.25$ (see Figures $F-1$ and 2 ) show that the maximal radial displacement is of the order of $6 \%$ of the maximal axial displacement $W(x)$. The maximum axial displacement deviates 
28

$0.1 \%$ from that of the infinite case near the ends. The maximal radial displacement varies by about $4 \%$ as $l / b$ varies from 1.25 to $\infty$ (see Figure $F-2$ ). The calculations also show that the choice of $R_{1}$ is unfortunate inasmuch as the eigenvalues become purely imaginary if $\mathrm{x}<0.7$ and $\nu$ are above some (not determined) value (possibly .35).

Appendix

$$
\begin{aligned}
& K=\int_{a}^{b}\left\{(\lambda+2 \mu)\left(\frac{d R_{1}}{d \xi}-\frac{R_{1}}{\xi}\right)^{2}-4 \mu \frac{d R_{1}}{d \xi} \frac{R_{1}}{\xi}\right\} \xi d \xi \\
& L=\int_{a}^{b} 4 \mu\left(\frac{d R_{2}}{d \xi}\right)^{2} \xi d \xi \quad M=\int_{a}^{b}(\lambda+2 \mu) b^{2} R_{2}^{2} \xi d \xi \\
& N=\int_{a}^{b} 4 \mu b^{2} R_{i}^{2} \xi d \xi \quad P=\int_{a}^{b} 8 \mu b R_{1} \frac{d R_{2}}{d \xi} \xi d \xi \\
& Q=\int_{a}^{b} 2 \lambda b\left(\frac{d R_{1}}{d \xi}+\frac{R_{1}}{\xi}\right) R_{2} \xi d \xi \\
& s=-2 \int_{a}^{b} \gamma b^{2} R_{2} \xi d \xi \\
& \theta=-2 \int_{a}^{b} b^{2} \Omega(\xi) R_{1} \xi d \xi \\
& J=-2 b x\left[R_{1}(x)\right] \\
& \begin{array}{c}
a_{1}=\frac{\bar{F}}{G_{1}} ; \quad a_{2}=\frac{\bar{H}}{G_{2}} ; \quad \quad a_{3}= \\
\bar{F}=\frac{P S}{2 L}
\end{array} \\
& G_{1}=F_{1} \cosh \lambda_{1} \ell+F_{2} G_{3} \cosh \lambda_{2} l \\
& G_{2}=H_{1} \cosh \lambda_{1} \ell+H_{2} G_{4} \cosh \lambda_{2} l \\
& G_{3}=-\frac{H_{1}}{H_{2}} \frac{\sinh \lambda_{1} l}{\sinh \lambda_{2} l} \\
& G_{4}=-\frac{F_{1}}{F_{2}} \frac{\sinh \lambda_{1} l}{\sinh \lambda_{2} l} \\
& H_{1,2}=2 M \alpha_{1,2}+Q \\
& F_{1,2}=2 N \lambda_{1,2}+P \alpha_{1,2} \\
& \lambda^{4}-\left[\frac{K}{N}+\frac{L}{M}-\frac{(P-Q)^{2}}{4 M N}\right] \lambda^{2}+\frac{K L}{M N}=0
\end{aligned}
$$



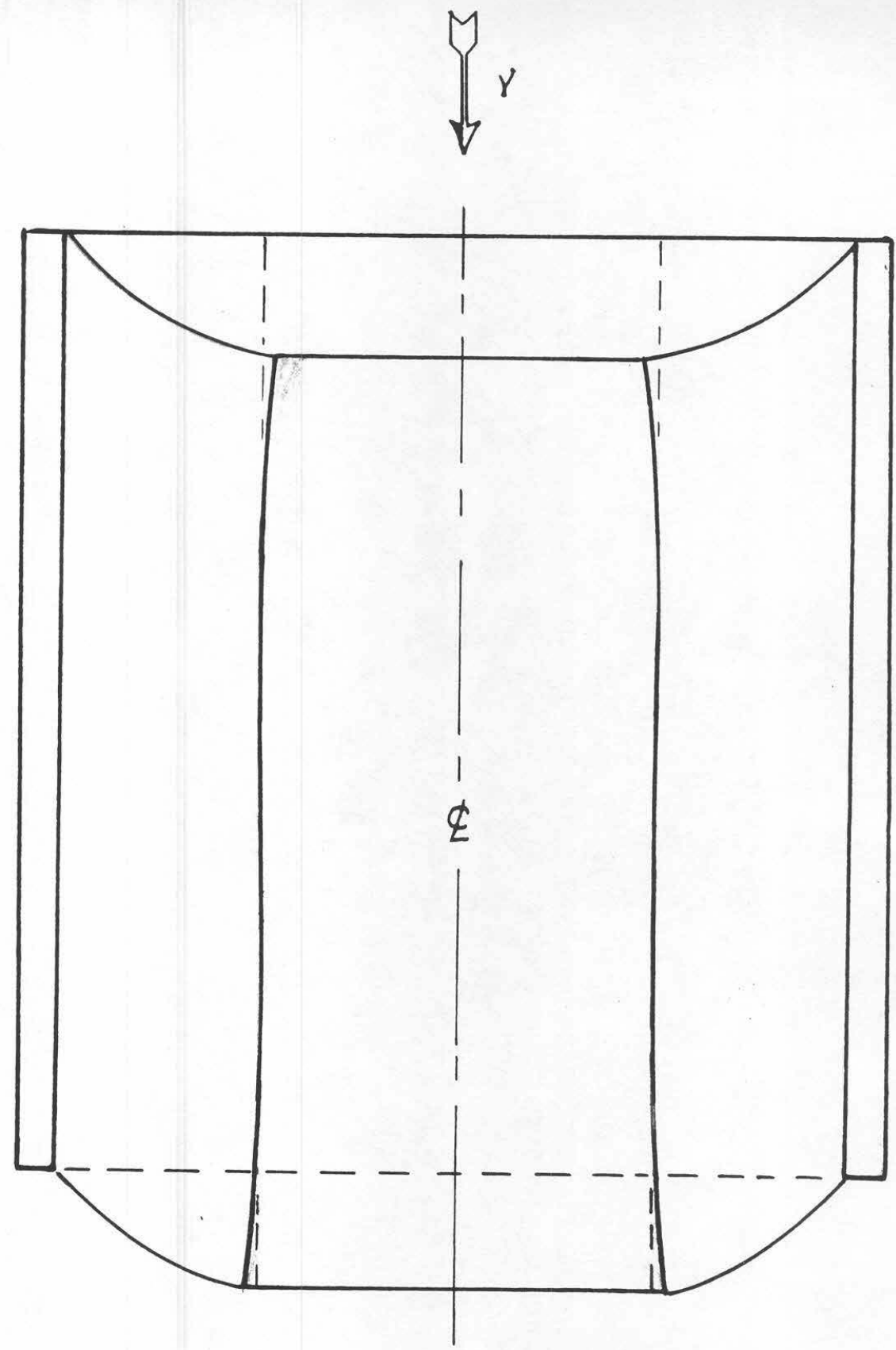

HOLLOW CYLINDER DEFORMATION

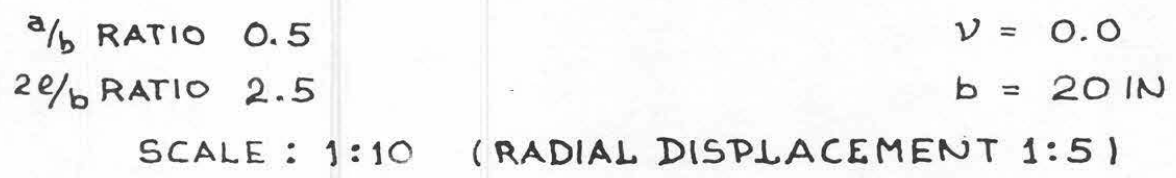

FIGURE $F-1$ 


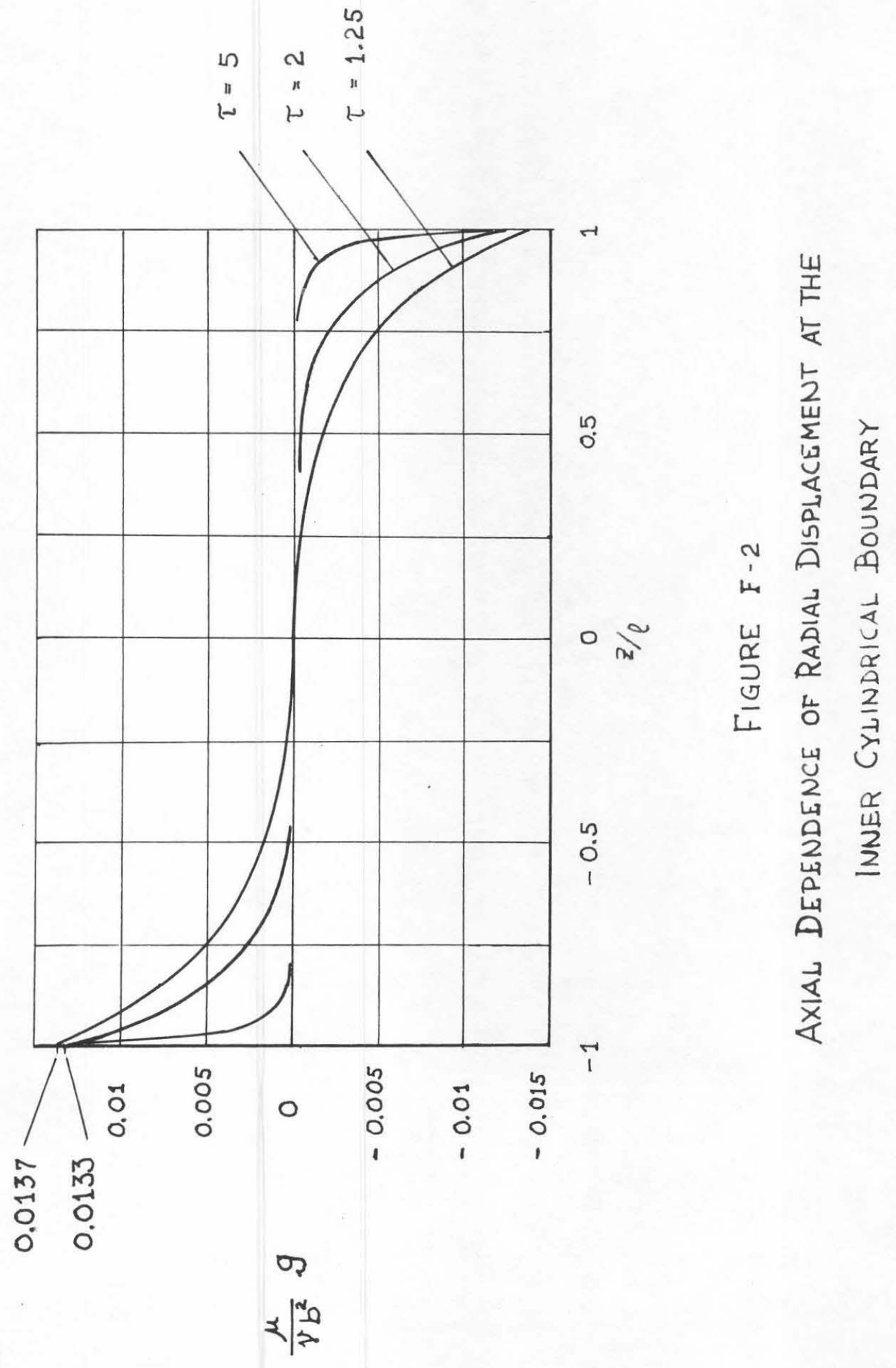


V。 FAILURE CRITERIA

Introduction

The viscoelastic analysis techniques provide a means of determining stress and strain, however a strength analysis is based, in addition, upon a criterion of failure. Unfortunately, failure criteria for solid propellant grains are in a very confused state and have been too confined to uniaxial testing. On the one hand, grain materials must withstand high short-time loads in contoured shapes, but not slump after long times. The first condition appears more as a stress dependency, whereas the second is a deformation dependency. In addition, it is not certain whether propellants fracture microscopically in shear or in normal tension; this being dependent upon the granular nature of the propellant. Double bases are more homogeneous and sensitive to plastisol content, whereas composites are highly sensitive to oxidizer content, fuel-to-osidizer binding strength, particle size and shape. Consequently, it is not yet certain as to the extent to which principle stress, strain, strain-rate, octahedral shear stress, shear strain, shear strain-rate, or strain energy enter the failure criterion.

Fortunately, $\operatorname{Smith}(10,11,12)$ has presented data which indicates a possible correlation between strain and strain-rate with the temperature compensation effect included. Figure 2 taken from Ref。10 is typical and is based on uniaxial, constant strain-rate data for GR-S rubber. $a_{T}$ is the temperature shift factor developed from the work of Williams, Landel, and Ferry ${ }^{(13)}$.

$$
\log a_{T}=\frac{-K_{1}\left(T-T_{5}\right)}{K_{2}+\left(T-T_{5}\right)}
$$

where $\mathrm{T}_{\mathrm{S}}$ is a reference temperature about $40^{\circ} \mathrm{C}$ above the glassy modulus temperature. It is interesting to note that the $\mathrm{K}_{1}$ and $\mathrm{K}_{2}$ constants are nearly universal for different polymers with the suitable glassy (or reference) temperatures given.

Since the Smith tests were conducted for uniaxial tension and a constant strain-rate, it needs to be extended to propellants under more 
general loading conditions. Different propellants have different physical properties which means testing of each separately to establish the existence of its corresponding Smith curve. In general, propellants may be expected to have more scatter in their fracturing results and may not superimpose as well upon shifting by the factor $\mathrm{a}_{\mathrm{T}}$. This introduces a need for a statistical measure or probability of failure, however differences in dependence on temperature can be retained (i。e, there is no a priori need to obtain a single curve as Smith did with polymers).

Biaxial and triaxial tests are required to obtain more realistic fracture behavior as it actually appears in a rocket design under load. Torsion of a hollow tube ${ }^{(14)}$ is a special biaxial test not too difficult to perform; the results of which can be compared directly with companion uniaxial tests which are at comparable strain-rates and temperatures. Several words of caution are given regarding these tests. Local strain measurements are most useful, although most tensile tests are conducted by measuring force versus crosshead displacement. An effective gage length must therefore be determined which is considerably longer, particularly in tension, than the physical gage length due to the tendency for the sample to creep through the grips. The increasing in effective gage length can be determined through a series of preliminary tests as a function of strain, strain-rate, and temperature by photographing incribed grids, dots or circles or by resistance measurements of lightly attached contacts. Then the main tests can have effective gage lengths attributed to them from charts made from the preliminary gage length tests.

An interesting series of tests are being initiated in which shear modulus and normal strain ratios are determined from tensile tests $(15$, 16, 17). This involves the photography of grids (dots and circles) to obtain both local axial elongation and local normal contraction. This does point out another problem; that of determining where and to what extent the specimen has necked down as failure is approached (true stress is based upon actual cross-section). In a similar way, the strain (and strain-rate) will vary over the length of the specimen, and these need to be known for development of a Smith curve. This problem of localized behavior is somewhat minimized (and compensated by using a known 
effective gage length) if strain at maximum stress is used rather than strain at break. After all, in a practical design, if this stress level is reached, then failure is bound to follow.

A series of tests carried out in conjunction with theoretical analysis for triaxial stress state in a thick walled cylinder bonded in a thin case will approach more closely the real rocket failure problem than simple tensile testing. This geometry is particularly favorable to analysis, both for internal pressurization and for thermal cycling. Some elastic solutions are indicated in the first two progress reports, and their extension to viscoelastic propellants is demonstrated by the examples in the previous section. The thermal problem is particularly simple if the ends are insulated against heat flow, since there is only one equation of equilibrium connecting the two unknown radial and tangential stresses. The second relation between these two quantities would be the failure criterion. Experiments might then be performed upon the cylinders at different temperatures and rates and compared with various failure hypotheses such as the modified Smith curve. Wise ${ }^{(18)}$ has started along these lines by exploring the normal strain hypothesis for one material and finds agreement with test data in the low temperature elastic range, but an investigation at the higher temperatures would be most desirable, particularly with a non-rigid case and non-bonded ends. This simple geometry can be extended to star configurations through the use of concentration factors under internal pressure presented by Ordahl and Williams $(19)$ and under thermal stresses by Williams $(20)$.

\section{Cumulative Damage Concept}

In any practical solid rocket design, account must be taken of the inter-related effects of the components and their environments。 The effect of the case on the grain, as demonstrated in section IV, is an example of this system design philosophy. In regard to the grain, the possibility of it cracking is dependent both upon its past environmental history and to the loading effects from adjacent "components." Therefore, it is required to solve the time and space dependent equations expressing the loading history. These viscoelastic solutions then 
produce the past histories of the localized regions of concern. In general, the localized rates of loading vary to a considerable extent, and hence we do not have a situation which matches the Smith failure curve which was developed at a number of different constant strainrates. This gives rise to a need for a cumulative damage concept that will permit flexibility in the strain-rate.

Miner ${ }^{(21)}$ verified the use of cumulative damage for aluminum in the $S-N$ fatigue tests. The concept was simply to add together the relative percentages of damage at each stress level (based on total number of cycles to damage at each stress level) until a hundred per cent damage was attained. Others ${ }^{(22)}$ have extended this concept to other metals and loading conditions in a more general (non-linear) way。

To reiterate by means of a specific illustration, if often happens that the usual fatigue criteria for metals of $\mathrm{N}$ cycles at a stress $\sigma *$ (Figure 1) is inappropriate. The usual reason is that in many applications one is concerned with a loading spectrum which is not constant. Hence while one may expect one million cycles before failure at say 50,000 psi stress, suppose the applied stress is halved to 25, 000 psi after only a half million cycles. The natural question then arises how many more cycles will the specimen withstand before failure? The usual approach has been to consider the problem from the standpoint of a cumulative damage. In the foregoing example one would say half the "life", say $\frac{1}{2} \mathrm{~N}_{50,000}$ ' had been used up when the stress was reduced. The specimen would then be expected to withstand half the life, i.e. $\frac{1}{2} \mathrm{~N}_{25,000}$, at the reduced stress. The total life for this particular assumed spectrum would therefore be $\frac{1}{2}\left(N_{50,000}+N_{25,000}\right)$. The general formulation for a spectrum of $M$ loadings $\sigma_{i}$, $(i=1,2,3 \ldots M)$, is

$$
\sum_{i=1}^{M}\left(\frac{N_{i}}{N_{R_{i}}}\right)^{n}=1
$$

where the life $L=\sum_{i=1}^{M} N_{i} \cdot N_{i}$ is the number of cycles at the 
stress $\sigma_{i}$, and $N_{R_{i}}$ is the number of cycles to rupture at the stress

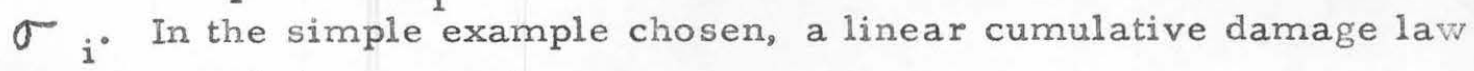
was assumed; hence $n=1$.

Based upon the preceding concept, it is proposed to inquire whether or not some similar law might be postulated for polymers, whose time dependence analogously to cycles is associated with strain rate. Smith has found a correlation between ultimate strain and strain rate, with temperature as a parameter (Figure 2), for specimens tested in uniaxial tension at a constant strain rate. For practical applications now, the strain rate is hardly ever constant during a test, particularly if the applied load or pressure is changing. It would therefore be desirable to be able to associate the failure of specimens subjected to. a varying strain rate to that at constant rate for which data, e.g. a Smith curve, has been obtained.

Suppose now that constant temperature conditions are assumed for simplicity, and write

$$
\sum_{i=1}^{M}\left(\frac{t_{i}}{t_{R_{i}}}\right)^{n}=1 \text { with } T=\sum_{i=1}^{M} t_{i}
$$

where $t_{i}$ and $t_{R_{i}}$ are the time the specimen is held at the strain rate $\dot{\epsilon}_{i}$ and the time to failure at $\dot{\epsilon}_{i}$ respectively. $T$ is the total time to rupture for the spectrum of strain rates $\dot{\epsilon}_{i}(i=1,2,3 \ldots . M)$. Passing now to an integral form of the linear cumulative failure law,

$$
\int_{0}^{T} \frac{d t(\dot{i})}{t_{R}(\dot{\epsilon})}=\hat{i}
$$

from which $T$ is to be determined for a specified spectrum $t(\dot{\epsilon})$ and Smith curve $t_{R}(\dot{\epsilon})$.

As a practical matter, it is often inconvenient to work with the times themselves. Actually, given

$$
\epsilon=\epsilon\left(x_{i}, t\right)
$$


from an analytical viscoelastic strain analysis, then by differentiation

$$
\dot{\epsilon}=\dot{\epsilon}\left(x_{i}, t\right)
$$

or inverting

$$
t=t\left(x_{i}, \dot{\epsilon}\right)
$$

alternate forms are possible. For the constant strain rate failure data (Figure 2), $\quad \epsilon_{\mathrm{R}}=\dot{\epsilon} t_{\mathrm{R}}$, so that

$$
t_{R}=\frac{\epsilon_{R}(\dot{\epsilon})}{\dot{\epsilon}}
$$

where an analytical representation of Figure $2, t_{R}=t_{R}(\dot{\xi})$, is required if numerical integration is to be avoided. The alternate scheme in (4) is to replace dt by $(\partial t / \partial \dot{\epsilon}) \partial \dot{\epsilon}$ and work completely in the rate space.

As an example to illustrate the mechanics of applying the postulate, assume to simplify the calculations that the actual strain response of the particular geometry under study is simple creep (Figure 3), viz

$$
\epsilon=\epsilon_{0}\left(1-e^{-t / \tau}\right)
$$

where $\epsilon_{0}$ is the initial strain. From (9) however the strain rate, by differentiation following (6), is

$$
\dot{\epsilon}=\frac{\epsilon_{0}}{\tau} e^{-t / \tau}=\dot{\epsilon}_{0} e^{-t / \tau}
$$

where $\dot{\epsilon}_{0}$ is the initial strain rate. Now by (7) one has

$$
t=\tau \log \left(\frac{\dot{\epsilon}_{0}}{\epsilon}\right)
$$

and 
where for physical significance in the approximation $\epsilon_{R}^{*} / \tau<\dot{\epsilon}_{0}$ of course.

\section{Discussion}

While certain liberties have been taken in approximating the failure data in order to demonstrate the means of carrying through the estimate of failure time, the steps are believed to affect the results in degree, not in principle. Indeed, given an actual viscoelastic strain analysis, the failure hypothesis could be tested by numerically integrating (4) using the actual Smith type data for the particular material involved.

There are, however, still fundamental points to be resolved: first, the form of the cumulative damage criterion, and second, its pertinence to combined, rather than solely uniaxial tensile, strain fields. With regard to the first, one might postulate an average value of $t(\dot{\epsilon}) / t_{R}(\dot{\epsilon})$ over the strain rate range $\dot{\epsilon}_{0}$ to $\dot{\epsilon}$ equaling unity。 Also S. R. Valluri has suggested a cumulative energy correlation based upon integrations of $\partial / \partial t\left(\frac{1}{2} \epsilon^{2}\right)$, inasmuch as he observes the Smith data straightens out quite well on $\log -\log$ paper when $\epsilon \dot{\epsilon}$ is plotted versus $t_{R}$ 。

In any event, should subsequent experiments establish that some sort of cumulative damage hypothesis has qualitative merit in simple examples, it would then be appropriate to attempt refinements in the form of non-linear cumulation, variable temperature situations, and combined stress or strain fields. 


$$
\frac{\partial t}{\partial \dot{\epsilon}}=-\frac{\tau}{\dot{\epsilon}}
$$

Turning now to the Smith curve, but replotting the data (Figure 4) in the form $t_{R}=t_{R}(\dot{\varepsilon})$ where the ultimate strains $\epsilon_{R}$ are indicated by hushmarks on the curve, one proceeds to curve fit the data. For illustrative purposes it is sufficient to approximate the actual Smith curve by the dashed line

$$
t_{R}=\frac{0.2}{\dot{\epsilon}}
$$

This form incidentally may be recognized as approximating the actual failure data by a constant rather than rate dependent ultimate strain -in this case $\epsilon_{\mathrm{R}}^{*}=0.2$.

With the assumed viscoelastic analysis yielding (11) and (12), and with an approximate failure threshhold given by (13), the cumulative damage postulation gives simply

$$
1=\int_{\dot{\epsilon}_{0}}^{\dot{\epsilon}(T)} \frac{1}{t_{R}(\dot{\epsilon})} \frac{\partial t}{\partial \dot{\epsilon}} d \dot{\epsilon}=\int_{\dot{\epsilon}}^{\dot{\epsilon}(T)} \frac{\dot{\epsilon}}{\epsilon_{R}^{*}}\left(-\frac{\tau}{\dot{\epsilon}}\right) d \dot{\epsilon}=-\left.\frac{\tau}{\epsilon_{R}^{*}} \dot{\epsilon}\right|_{\dot{\epsilon}_{0}} ^{\dot{\epsilon}(T)}
$$

and hence

$$
\frac{\epsilon_{R}^{*}}{\tau}=\dot{\epsilon}_{0}-\dot{\epsilon}(T)=\dot{\epsilon}_{0}\left(1-e^{-T / \tau}\right)
$$

so that failure would be anticipated when the initial strain rate $\dot{\epsilon}_{0} \equiv \epsilon_{0} / \tau$ is reduced by the amount $\epsilon_{\mathrm{R}}^{*} / \tau$. For a $\tau$, the characteristic relaxation time, of say 1 second and $\epsilon_{R}^{*}=0.2$, failure should occur when the initial strain rate is reduced by $0.2 \mathrm{in} / \mathrm{in} / \mathrm{sec}$.

The life is then found to be proportional to the relaxation time, viz

$$
T=\tau \log \left(\frac{1}{1-\frac{\epsilon_{R}^{*} \tau}{\dot{\epsilon}_{0}}}\right)
$$




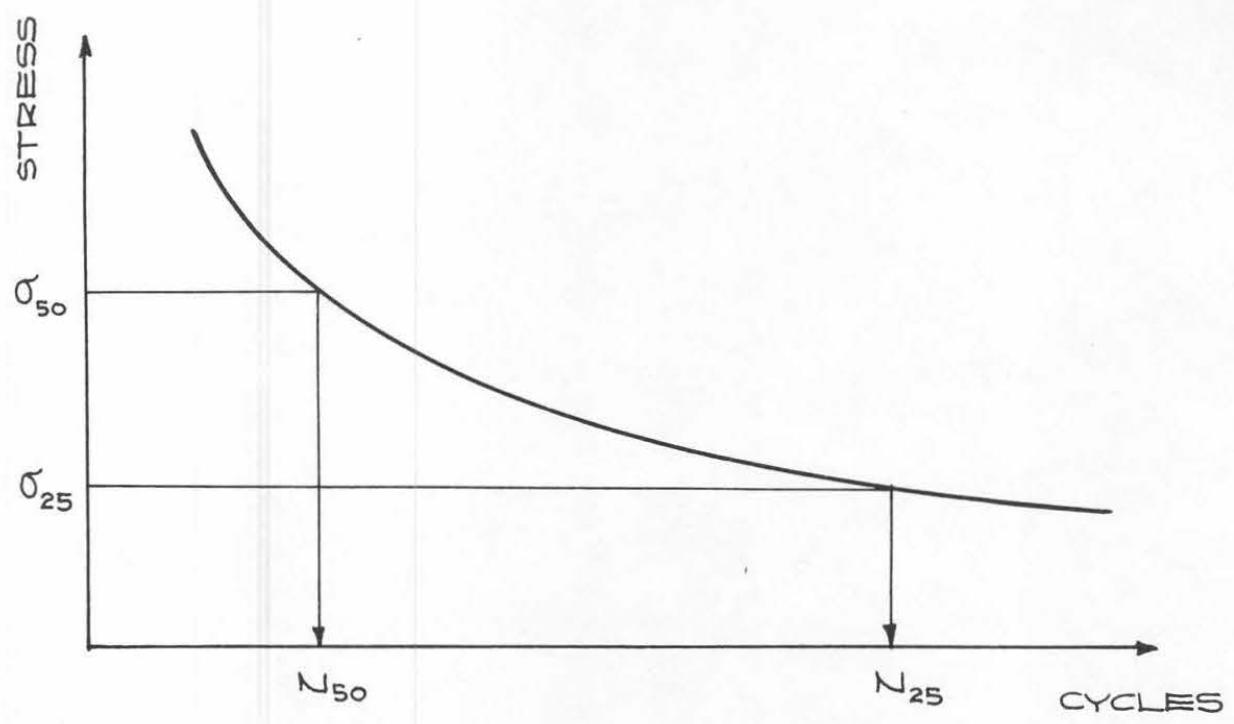

FIG. 1 S-N FATIGUE CURVE

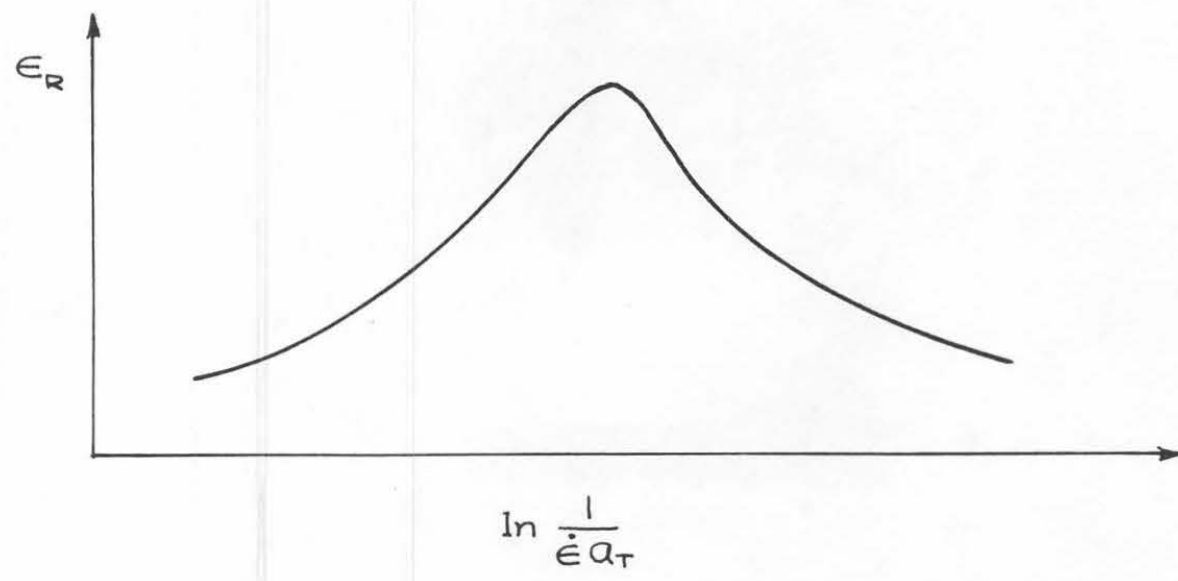

FIG.2 SMITH FAILURE CURVE FOR POLYMERS REF.: JPL MEMORAMDUM NO. 20-178 


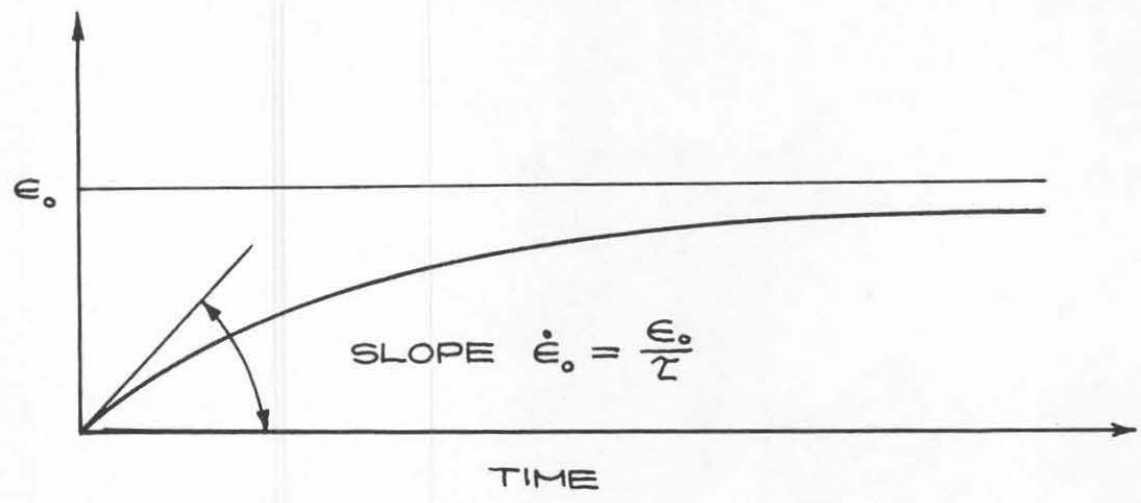

FIG. 3 CREEP CURVE

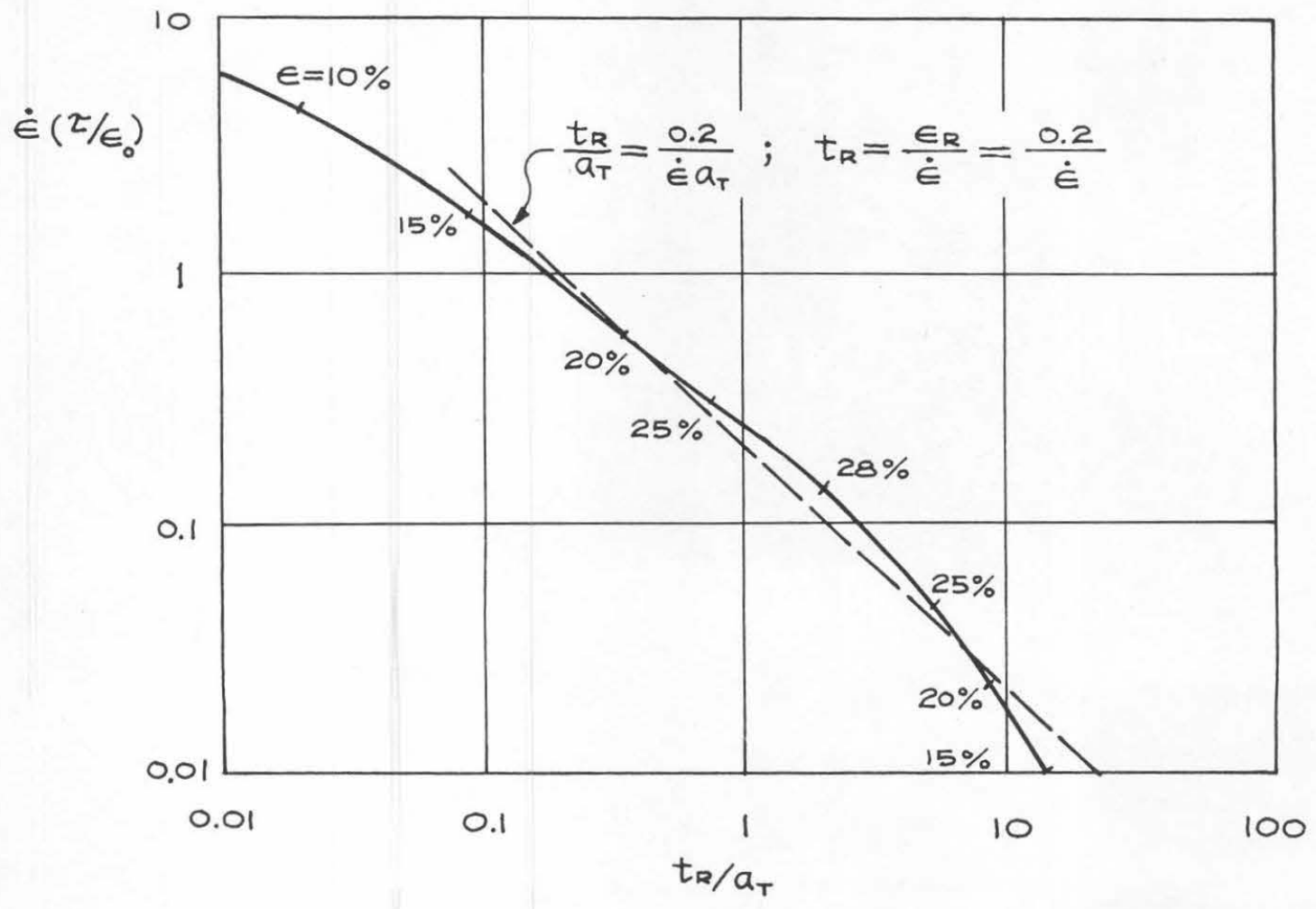

FIG. 4 MODIFIED SMITH CLRVE 


\section{REFERENCES}

1. R. A. Schapery, L. D. Stimpson, M。 L. Williams, "Fundamental Studies Relating to Systems Analysis of Solid Propellants", California Institute of Technology, GALCIT 101, Progress Report No. 1, January 15, 1959.

2. R. A. Schapery, L. D. Stimpson, M. L. Williams, "Fundamental Studies Relating to Systems Analysis of Solid Propellants", California Institute of Technology, GALCIT 101, Progress Report No. 2, April 15, 1959.

3. M. A. Biot, "Linear Thermodynamics and the Mechanics of Solids", Proc of the Third U. S. Nati. Congro of Appl. Mechs., Brown University, American Soc。 of Mech. Engrs., June 11-14, 1958, pp.15-16。

4. J. D. Clem, W.H.Groetzinger, J. E. Johnson, C. H. Parr, Quarterly Progress Report on Weapons Research, Rohm and Haas Co., Rept. No.P-57-5, April 10, 1957.

5. P. L. Nichols, "Control of Chemical and Physical Factors in the Application of Casting Resins", Soc. of Plastics Engrs. Journ., November 1956.

6. P. L. Nichols, A. G. Presson, "Heat-T ransfer Calculations Related to Solid-Propellant Curing", California Institute of Technology, Jet Propulsion Laboratory, Rept. No。20-68, September 17, 1953.

7. H. S. Carslaw, J.C. Jaeger, "Conduction of Heat in Solids", Oxford, Clarendon Press, sections 79, 126, and 137, 1947.

8. E. Jahnke, F. Emde, "Tables of Functions", 4th Ed., Dover Publications, 1945, pp.205-206. 
9. W. G. Knauss, "Elastic Displacements in a Hollow, Circular Cylinder of Finite Length, Rigidly Supported on its Outer Surface and Subjected to Internal Pressure, Radial and Axial Acceleration", California Institute of Technology, GALCIT SM 59-9, May 1959.

10. T。L. Smith, "Elastomeric-Binder and Mechanical Property Requirements for Solid Propellants", California Institute of Technology, Jet Propulsion Laboratory Memo. No。20-178, January $7,1959$.

11. T. L. Smith, "Dependence of the Ultimate Properties of a GR-S Rubber on Strain Rate and Temperature", Journ. Polymer Sci., pp. 99-113, October 1958.

12. T. L. Smith, "Viscoelastic Behavior of Polyisobutylene under Constant Rates of Elongation", Journ. Polymer Sci., pp。39-100, April 1956.

13. M. L. Williams, R. F. Landel, J.D. Ferry, "The Temperature Dependence of Relaxation Mechanisms in Amorphous Polymers and Other Glass-Forming Liquids", Journ. American Chem. Soc., 77: $3701-3707,1955$.

14. W. Dowler, G. Lewis, L。D. Stimpson, "Torsion Testing of Solid Propellants", California Institute of Technology, Jet Propulsion Laboratory Publ. No。30-4, April 24, 1959.

15. K. Bischel, "Propellant Property Data Being Measured at the Naval Ordnance Test Station", Bull. of the 17th Mtg。 of the JANAF Panel on Physical Properties of Solid Propellants, May 1958, pp. 203-225。

16. P. J. Blatz, "Grain Material Representation by Models", JANAF Symposium on Structural Analysis of Solid Propellant Grains, Washington, D.C., June 16, 195.9. 
17. L. D. Stimpson, "Shear Modulus and Normal Strain Ratio Determined from Tensile Tests on Viscoelastic Materials", California Institute of Technology, GALCIT SM 59-15, July 1959.

18. J. S. Wise, "The Prediction of Limiting Pressures in Symmetrical Axially-Uniform, Solid Propellant Configurations", Proc。of 13th JANAF Solid Propellant Group, Chicago, Ill., May 1957.

19. D. D. Ordahl, M. L. Williams, "Preliminary Photoelastic Design Data for Stresses in Rocket Grains", Jet Propulsion, June 1957.

20. M. L. Williams, "Some Thermal Stress Design Data for Rocket Grains", American Rocket Soc。Journ。, April 1959.

21. M。A. Miner, "Cumulative Damage in Fatigue", Journ。Appl. Mechs, pp. A-159-164, September 1945.

22. A. M. Freudenthal, R.A. Heller, "Cumulative Fatigue Damage of Aircraft Structural Materials, Part 2: 2024 and 7075 Aluminum Alloy Additional Data and Evaluation", Columbia University, WADC Tech。Note 55-273, Part 2, October 1956. 